\title{
Análisis de la Provisión Fondo de Innovación para la Competitividad: fundamento, operación y espacios de mejora
}

\author{
Analysis of the Provisión Fondo de Innovación para la \\ Competitividad: foundation, operation and improvement spaces
}

Rodrigo Candia ${ }^{a}$; Francisca Del Fierro ${ }^{b}$, Juan Miranda ${ }^{c}$

a Unidad de Fortalecimiento para la Descentralización, Subsecretaría de Desarrollo Regional, Gobierno de Chile. rodrigo.candia@subere.gov.cl

${ }^{b}$ Departamento de Gestión de Inversiones Regionales, Subsecretaría de Desarrollo Regional, Gobierno de Chile. francisca.delfierro@subdere.gov.cl

c Departamento de Gestión de Inversiones Regionales, Subsecretaría de Desarrollo Regional, Gobierno de Chile. juan.miranda@subdere.gov.cl

\section{RESUMEN}

El modelo de desarrollo económico de Chile se ha caracterizado por estar sustentado principalmente en la explotación de commodities (principalmente de los sectores minero y agrícola). A partir de él, entre los años 1990 y 1999, se obtuvo una tasa de crecimiento promedio de 6,4\% (a pesar de la recesión que provocó la crisis asiática), no obstante, entre los años 2000 y 2010, ese crecimiento alcanzó solo un 3,8\% promedio.

Esta situación, según diversos diagnósticos, refleja un agotamiento del modelo señalado, lo que ha derivado en la necesidad de introducir cambios en él. En ese contexto, es que se crea el Fondo de Innovación para la Competitividad el año 2006, con el fin de focalizar recursos para promover la generación de una economía asentada en el conocimiento con fuente de diversificación y sofisticación.

Luego, el 2008 se crea la provisión Fondo de Innovación para la Competitividad de asignación regional, entregándose su administración a los gobiernos regionales con el propósito de apoyar el desarrollo económico territorial mediante la innovación regional y el mejoramiento de su competitividad.

$\mathrm{Al}$ respecto, existen diversas críticas en relación al cumplimiento de sus objetivos, a los resultados obtenidos y a la forma en que opera su asignación, las cuales cobran más importancia en un contexto de necesidad de mejorar la productividad y de demandas por mayor descentralización, siendo el ámbito fiscal uno de los más relevantes.

Es por lo expuesto que este trabajo busca, a partir de la revisión de literatura relacionada a políticas de ciencia, tecnología e innovación, así como también del análisis descriptivo de bases de datos sobre de la distribución y asignación del FIC y de la aplicación de una encuesta de percepción respecto a su justificación y funcionamiento, extraer antecedentes que sean de utilidad para evaluar el desempeño de esta provisión y plantear recomendaciones que permitan su mejoramiento dentro de los temas ya señalados. Con base en lo anterior, se realizan propuestas en relación a definiciones de política y de operación de la provisión FIC, las cuales serán de utilidad para apoyar el trabajo que realiza SUBDERE en la materia.

Palabras clave: Innovación; desarrollo regional; competitividad territorial 


\section{ABSTRACT}

Chile's economic development model has been characterized by being mainly based on the exploitation of commodities (primarily from the mining and agricultural sectors). From it, between the years 1990 and 1999, an average growth rate of $6.4 \%$ was obtained (despite the recession caused by the Asian crisis), however, between 2000 and 2010, that growth reached only $3.8 \%$ on average.

This situation, according to various diagnoses, reflects a depletion of the aforementioned model, which has resulted in the need to introduce changes in it. In this context, the Innovation Fund for Competitiveness is created in 2006, in order to focus resources to promote the generation of an economy based on knowledge with a source of diversification and sophistication.

Then, in 2008, the provision of the Innovation Fund for the Competitiveness of regional allocation was created, giving its administration to the regional governments with the purpose of supporting territorial economic development through regional innovation and improving its competitiveness.

In this regard, there are several criticisms regarding the fulfillment of its objectives, the results obtained and the way in which its allocation operates, which become more important in a context of the need to improve productivity and demands for greater decentralization, being the Fiscal scope one of the most relevant.

It is for this reason that this work seeks, from the review of literature related to science, technology and innovation policies, as well as the descriptive analysis of databases on the distribution and allocation of the FIC and the application of a survey of perception regarding its justification and operation, to detect antecedents that are useful for evaluating the performance of this provision and make recommendations that evaluate its improvement within the topics indicated. Based on the above, proposals will be made in relation to the policies and operation of the FIC provision, which will be useful to support the work carried out by SUBDERE in this area.

Key words: innovation, regional development, territorial competitiveness.

\section{INTRODUCCIÓN}

El 2008 se crea la provisión Fondo de Innovación para la Competitividad (FIC$\mathrm{R}$ o provisión FIC), entregándose a los gobiernos regionales con el propósito de apoyar el desarrollo económico territorial mediante la innovación regional y el mejoramiento de su competitividad. Esta provisión opera financiando instrumentos de agencias como la Corporación de Fomento de la Producción (CORFO), la Comisión Nacional de Investigación Científica y Tecnológica (CONICYT) y otras y, además, proyectos específicos de universidades, institutos y centros de Investigación y Desarrollo (I+D).

Al respecto, existen diversas críticas en relación al cumplimiento de sus objetivos, a los resultados obtenidos y a la forma en que opera su asignación, las cuales cobran más importancia dada la necesidad de mejorar la productividad y de demandas por mayor descentralización, siendo elámbito fiscal uno de los más relevantes.

Es por lo expuesto que este trabajo busca contar con información y análisis que sean de utilidad para evaluar el desempeño de esta provisión y plantear recomendaciones que permitan su mejoramiento dentro de los temas ya señalados. A partir de ello, se espera realizar propuestas en relación a definiciones de política y de operación de la provisión FIC, que sean de utilidad para apoyar el trabajo que 
realiza la Subsecretaría de Desarrollo Regional y Administrativo (SUBDERE) en la materia.

$\mathrm{Su}$ estructura es la siguiente: en la primera parte, a partir de revisión bibliográfica, se realiza una recopilación de los principales planteamientos que hoy en día existen en relación a políticas regionales de innovación y desarrollo industrial (como fundamentos asociados a la existencia de un fondo como el que se analiza en este trabajo), así como también algunos datos que permiten contextualizar la situación y evolución que ha tenido el país y sus regiones en ambos marcos. Luego se ahonda en la situación de la provisión FIC, analizando aspectos legales y operativos a partir de la revisión de la normativa que regula el funcionamiento de esta fuente de financiamiento y de documentos tales como la evaluación que realizó la Dirección de Presupuestos (DIPRES) del FIC-R el año 2011 (entre otros antecedentes que dispone SUBDERE), lo que se complementa con un análisis principalmente descriptivo de la asignación de los recursos por institución receptora, a partir de los datos provistos por los gobiernos regionales el año 2017. Posteriormente, se entregan los resultados y análisis efectuados a partir de la encuesta realizada por SUBDERE ese mismo año en materia de percepción del funcionamiento del FIC-R (la que fue elaborada considerando los antecedentes analizados anteriormente para identificar los ámbitos relevantes de preguntar), la que estuvo dirigida a los distintos actores que influyen en su operación, así como también del taller de trabajo realizado en ese periodo, en donde se invitaron a representantes de instituciones receptoras y a otros actores relevantes para discutir sobre el desempeño del FIC-Ra partir de los datos y análisis anteriormente señalados.

Con base en todo ello, finalmente se entregan algunas conclusiones y recomendaciones, las cuales están focalizadas principalmente en mejorar aspectos estratégicos y operativos de esta fuente de financiamiento.

\section{CONTEXTO DE POLÍTICA}

De acuerdo con Eyzaguirre, Marcel, Rodríguez y Tokman (2005), el contexto general del país que justifica la necesidad de avanzar hacia una economía basada en el conocimiento y que, a su vez, derivó en la conformación de un Sistema Nacional de Innovación y del Fondo de Innovación para la Competitividad, creado el 2006, y luego en la creación de la provisión FIC en el año 2008; se caracteriza por el agotamiento del modelo de desarrollo basado en la explotación y exportación de materias primas (que en Chile representan el $85 \%$ del total de exportaciones, mientras que en países desarrollados esa participación llega al 25\%), lo que se ha manifestado en un deterioro de los niveles de crecimiento de la economía y de la productividad total de factores (PTF), entre otros indicadores.

Esto se complementa con lo expuesto por Lauterbach (2015) quien, a partir de análisis propios como de trabajos realizados por Ricardo Haussman y otros, revela que la matriz chilena de exportaciones está compuesta por producto de poco valor agregado (poco uso de conocimiento y de capital humano avanzado) que además son producidos por otros países.

Para abordar esa situación se requiere, siguiendo a Eyzaguirre et al. (op. cit.), la generación y aprovechamiento del conocimiento que permita pasar de una economía basada en ventajas comparativas estáticas a otra sustentada en ventajas competitivas dinámicas (economía del conocimiento). Ello por cuanto las ventajas 
comparativas estáticas son vulnerables, especialmente aquellas basadas en recursos naturales (como ya pasó, por ejemplo, con la explotación del Salitre).

En cuanto a la estrategia a seguir, los autores señalados (op. cit.) plantean algunos ejes que están directamente relacionados con los temas actuales que forman parte de la discusión que se está dando actualmente en materia de políticas regionales de desarrollo industrial y de innovación y que fueron presentadas anteriormente, los que dicen relación con el aprovechamiento "inteligente" de los recursos naturales para la sofisticación y diversificación de la producción. Estos ejes son los siguientes:

- Capital humano.

- Capacidad de innovación.

- Marco institucional y de incentivos económicos, lo cual contempla estabilidad macroeconómica, regulación clara y buen funcionamiento de mercado de capitales.

Por su parte, a través del mismo documento también se entregan algunas características deseables que debieran lograrse como parte de un entorno proclive a la economía del conocimiento, siguiendo la experiencia internacional. Tales características son las siguientes:

- Es necesario que las políticas de innovación estén asociadas a ventajas comparativas que existan efectivamente, lo cual es determinado por el mercado. Esto es importante para evitar la tentación de promover el desarrollo de sectores para los que no existen condiciones que permitan vislumbrar que tendrán una probabilidad razonable de éxito.

- Es necesario promover los encadenamientos hacia atrás y hacia los costados (sectores complementarios) de los sectores exportadores, como forma de potenciación conjunta y de maximización del potencial exportador.

- El sector privado debe fin anciar la mayor parte del gasto que el país hace en $\mathrm{I}+\mathrm{D}$.

A estas características se pueden agregar otras planteadas por Crespi, Fernández-Arias y Stein (2014), a partir del reconocimiento de la complejidad que representan las políticas de innovación debido a las fallas de mercado que deben abordar, la multiplicidad de actores e intereses ligados a ellas y al largo periodo que requieren para su maduración y obtención de resultados; como las siguientes:

- Necesidad de contar con capacidades institucionales para la coordinación, implementación y monitoreo y evaluación de políticas.

- Orientar la acción a partir estrategias de largo plazo consensuadas entre actores públicos, privados y académicos.

A ellas, los autores citados agregan algunos componentes obtenidos de la experiencia para la definición de políticas exitosas de innovación. Entre estos, destaca la existencia de consensos público-privados con alcance de largo plazo respecto a la importancia del tema y al rol que a cada uno de ellos le corresponde; la focalización de políticas iniciales en la adopción de tecnologías extranjeras y generación de condiciones para la innovación (infraestructura y capital humano), lo que debe complementarse con investigación aplicada a los sectores productivos clave, lo que no necesariamente trae consigo un fuerte sistema de protección intelectual; la importancia que tiene la generación de un entorno macroeconómico 
adecuado para el desarrollo de la actividad, sumado a un creciente fomento a la inversión en ciencia y tecnología e innovación empresarial; la disposición de un abanico de instrumentos públicos para la innovación y aspectos relacionados a ella y; la generación de capacidades institucionales para el diseño implementación, evaluación y monitoreo de la política y sus instrumentos, que permitan el aprendizaje y mejoramiento continuo.

Dentro de las modalidades de intervención que tiene a su disposición el sector público, una de las principales son los incentivos fiscales los cuales, de acuerdo con Crespi et al. (op. cit.), se manifiestan a través de subsidios o de incentivos tributarios. $\mathrm{Su}$ objetivo, siguiendo a los autores citados, es abordar las fallas de mercado señaladas anteriormente reduciendo el costo de capital para realizar innovación, así como también para promover conductas deseables, tales como la colaboración público-privado o privado-académica, entre otras.

En el caso del FIC, esta fuente de financiamiento opera mediante la entrega de subvenciones concentradas en entidades de investigación y empresas, tal como se expondrá más adelante. En ese sentido, Crespi et al. (op. cit., p. 88) entregan algunas recomendaciones para su buen diseño:

- "Implementación de un proceso de asignación competitivo (llamada a concurso).

- La tasa de subsidio es proporcional al tamaño de las externalidades (más altas en los bienes públicos, investigación genérica o proyectos de colaboración).

- Asignación transparente mediante un consejo público-privado basándose en evaluaciones de revisores inter pares externos e independientes.

- Creación de capacidades en las empresas para la formulación de proyectos y estableciendo objetivos para el financiamiento.

- Inclusión de una cláusula de expiración con un riguroso sistema de monitoreo y evaluación".

Junto con ello, los autores señalados (op. cit) destacan la forma en que la política de innovación puede hacerse cargo de dos de las fallas de mercado asociadas, que son el riesgo moral y la captura. En ese sentido, señalan que "[...] estos problemas se pueden minimizar cuando se aplican diversos principios de diseño básicos (como el diseño de programas con cláusula de expiración, el cofinanciamiento por parte del sector privado, la asignación mediante procesos competitivos, la evaluación ex ante mediante revisores de pares externos y evaluaciones de impacto ex post)" (op. cit., p. 111-112).

Por otro lado, es importante considerar la situación que presenta el país en materia de $\mathrm{I}+\mathrm{D}$, entendiendo que esta actividad es una de las principales fuentes para el desarrollo de innovación basada en conocimiento. Al respecto, el panorama general es el que se presenta en el Cuadro 1. Este muestra la composición general del gasto en I+D por sector y la dinámica que ha tenido entre los años 2008 y 2014, reflejando la participación importante que tiene el Estado en la materia y la baja que ha tenido la participación de las empresas, lo cual no es deseable si se compara con patrones de países desarrollados, en donde el mayor gasto en I+D proviene precisamente de ellos. 
Cuadro 1. Síntesis de datos de la situación del país en materia de I+D.

\begin{tabular}{|l|l|r|r|}
\hline \multicolumn{2}{|c|}{ Indicadores } & 2008 & 2014 \\
\hline \multirow{3}{*}{$\begin{array}{l}\text { \% participación en la } \\
\text { inversión en I+D }\end{array}$} & Estado & $34 \%$ & $44 \%$ \\
\cline { 2 - 4 } & Empresas & $44 \%$ & $32 \%$ \\
\cline { 2 - 4 } & Educación superior & $17 \%$ & $9 \%$ \\
\cline { 2 - 4 } & IPSFL $^{1}$ & $2 \%$ & $1 \%$ \\
\cline { 2 - 4 } & Fondos internacionales $^{-}$ & $3 \%$ & $14 \%$ \\
\hline $\begin{array}{l}\text { Gasto en I+D como porcentaje del Producto Interno } \\
\text { Bruto (PIB) }\end{array}$ & $0,4 \%$ & $0,38 \%$ \\
\hline
\end{tabular}

Fuente: elaboración propia con base en Ministerio de Economía (2010) y Ministerio de Economía (2016a).

A esto se pueden sumar datos a nivel regional, los cuales se incluyen en los Cuadros 2 y 3 :

Cuadro 2. Participación porcentual en el gasto en actividades innovativas, según región de ejecución (2009-2010 y 2013-2014).

\begin{tabular}{|l|r|r|r|r|}
\hline \multicolumn{1}{|c|}{ Regiones } & 2009 & \multicolumn{1}{c|}{2010} & 2013 & 2014 \\
\hline Arica y Parinacota & 1,1 & 1 & 0,8 & 0,8 \\
\hline Tarapacá & 2,9 & 2,8 & 1,6 & 0,8 \\
\hline Antofagasta & 4,4 & 4,4 & 3,3 & 4,9 \\
\hline Atacama & 1,7 & 2,2 & 1,1 & 0,9 \\
\hline Coquimbo & 1,4 & 1,6 & 7,7 & 5,1 \\
\hline Valparaíso & 8,8 & 9,6 & 10,3 & 9,1 \\
\hline Metropolitana & 43,6 & 45,7 & 42,3 & 45,3 \\
\hline O'Higgins & 3 & 2,9 & 8,7 & 7,6 \\
\hline Maule & 3,9 & 2,2 & 6,2 & 5,6 \\
\hline Biobío & 10,5 & 9,8 & 7,1 & 4,8 \\
\hline La Araucanía & 7,3 & 6,3 & 3,4 & 4,4 \\
\hline Los Ríos & 2,1 & 2,5 & 2,2 & 2,3 \\
\hline Los Lagos & 6,1 & 5,9 & 2,9 & 4,5 \\
\hline Aysén & 0,6 & 0,7 & 0,9 & 1,2 \\
\hline Magallanes & 2,6 & 2,5 & 1,3 & 2 \\
\hline Total & 100 & 100 & 100 & 100 \\
\hline
\end{tabular}

Fuente: elaboración propia con base en Ministerio de Economía (2016b) y Ministerio de Economía (2012).

Tal como se puede apreciar en el cuadro anterior, la inversión en innovación está fuertemente concentrado en la región Metropolitana, la que está muy por sobre el resto de las regiones del país. Luego, destacan un grupo de regiones que incluye a Valparaíso, Coquimbo, O’Higgins, Maule, Antofagasta y Biobío, llamando la atención en especial el caso de la región de O'Higgins, la que hasta se poco no contaba

${ }^{1}$ IPSFL: Instituciones Privadas sin fines de Lucro. 
con universidades con su casa central en la zona, y Biobío, que tiene una estructura de universidades similar a la región de Valparaíso.

Cuadro 3. Distribución del esfuerzo investigador por regiones².

\begin{tabular}{|l|r|r|}
\hline Regiones & 2003 & 2013 \\
\hline Arica y Parinacota & 0,57 & 1,68 \\
\hline Tarapacá & 0,57 & 0,57 \\
\hline Antofagasta & 3,66 & 3,53 \\
\hline Atacama & 0,09 & 0,25 \\
\hline Coquimbo & 2,81 & 2,72 \\
\hline Valparaíso & 6,19 & 11,0 \\
\hline Metropolitana & 62,59 & 53,36 \\
\hline O'Higgins & 0,09 & 0,10 \\
\hline Maule & 1,34 & 3,43 \\
\hline Biobío & 12,84 & 12,17 \\
\hline La Araucanía & 2,27 & 4,62 \\
\hline Los Ríos & 5,8 & 4,8 \\
\hline Los Lagos & 0,74 & 1,04 \\
\hline Aysén & 0,06 & 0,26 \\
\hline Magallanes & 0,4 & 0,49 \\
\hline Total & 100 & 100 \\
\hline
\end{tabular}

Fuente: CONICYT (2015).

Lo analizado anteriormente también tiene relación con lo que muestra el Cuadro 3, en donde destaca la actividad científica realizada por región (de lo que se puede derivar la capacidad que tienen al respecto), al mostrar nuevamente la alta concentración en la región Metropolitana, así como también la importante participación que tienen regiones tales como Valparaíso y Biobío y la baja que tiene la de O’Higgins.

\section{ANTECEDENTES GENERALES DE LA PROVISIÓN FONDO DE INNOVACIÓN PARA LA COMPETITIVIDAD}

De acuerdo a lo señalado en la evaluación efectuada a la provisión FIC por parte de la Dirección de Presupuestos (DIPRES, 2011), esta fuente de financiamiento se creó el año 2008 con el fin de "contribuir al desarrollo económico de los territorios, promoviendo la innovación regional y el aumento de la competitividad" (op. cit.: P. 18), siendo su propósito el contar con "sistemas regionales de innovación más eficientes para promover el desarrollo en las regiones" (op. cit.: P. 19).

De acuerdo con Balbontín, Roeschmann y Zahler (2018), esta fuente de financiamiento representó un $7 \%$ del total del presupuesto en que invierte el Gobierno Central en ciencia, tecnología e innovación, para el año 2017, y un 7\% en promedio entre los años 2008 y 2017. Además, destacan que el importante aumento

${ }^{2}$ Mide la distribución porcentual por regiones de la actividad científica realizada en el país. 
que han tenido en el periodo 2008-2018, llegando a un 65\%, así como también que representó para el año 2017 un $4 \%$ del presupuesto total de los gobiernos regionales ${ }^{3}$.

Su distribución, entre los años 2008 y 2012, se efectuó a través de resoluciones emitidas por la SUBDERE elaboradas con base en la propuesta de distribución formulada por el Ministerio de Economía la cual, a su vez, era definida a partir de los siguientes criterios (SUBDERE, 2011): 60\% para las llamadas "regiones mineras", entendiendo como tales a aquellas cuyo PIB minero regionales represente más de un 2,5\% del PIB minero nacional (excluyendo al petróleo y al gas) y su PIB regional.

Dentro de ellas, el porcentaje señalado se distribuye en un 50\% de manera uniforme; 30\% siguiendo el criterio de porcentajes reponderados para ellas de la forma en la que se distribuye el FNDR ${ }^{4}$ (artículo 76 de la Ley Orgánica Constitucional de Gobierno y Administración Regional -19.175); y 20\% respecto a su participación relativa en el total del PIB minero del grupo de regiones que se incluye dentro de esta categoría.

El 40\% restante se distribuye entre las regiones no consideradas dentro del criterio anterior de la siguiente forma: 50\% por igual entre todas ellas; y 50\% de acuerdo al criterio de porcentajes reponderados para este conjunto de la forma en la que se distribuye el FNDR (siguiendo el artículo ya citado).

Posteriormente, desde el año 2013 hasta el actual se distribuyeron los recursos directamente dentro de los presupuestos de inversión de cada gobierno regional, como "Fondos FIC (sin distribuir)" siguiendo los criterios señalados, lo cual permitió reducir el tiempo asociado a la disponibilidad de información por parte de los gobiernos regionales respecto al monto que le corresponde en cada año.

En cuanto al procedimiento de operación de la provisión, que está regulado en las resoluciones $\mathrm{N}^{\circ} 277 / 2011$ y No2/2016 de SUBDERE, este se resume en la Figura 1. A través de estos recursos se pueden abordar las líneas de financiamiento descritas en el Cuadro 4.

3 Para complementar lo señalado, los autores citados señalan que "El presupuesto del Gobierno Central en ciencia, tecnología e innovación (CTI) para el año 2017 es de \$644.730 millones, representando un 0,36\% del PIB y un 1,5\% del total de la Ley de Presupuestos” (op. Cit, p.42).

4 FNDR: Fondo Nacional de Desarrollo Regional. 


\section{Figura 1. Operación general de la provisión FIC 5 .}

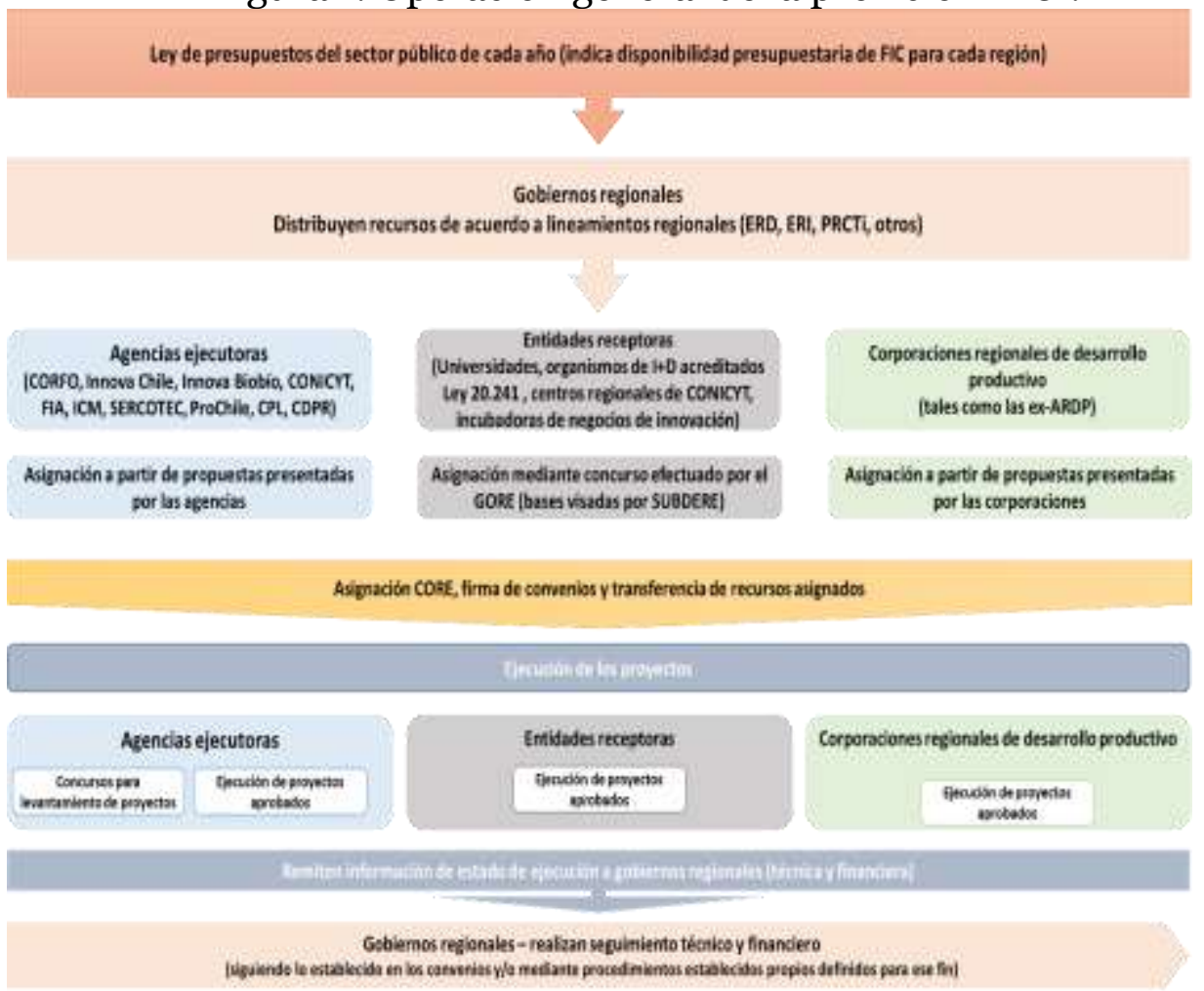

Fuente: elaboración propia con base en SUBDERE, 2011 y en SUBDERE, 2016.

5 ERD: Estrategia Regional de Desarrollo; ERI: Estrategia Regional de Innovación; PRCTI: Política Regional de Ciencia, Tecnología e Innovación; CORFO: Corporación de Fomento de la Producción; CONICYT: Comisión Nacional de Investigación Científica y Tecnológica; FIA: Fundación para la Innovación Agraria; ICM: Iniciativa Científica Milenio; SERCOTEC: Servicio de Cooperación Técnica; CPL: Consejo Nacional de Producción Limpia; CDPR: Comité de Desarrollo Productivo Regional; ARDP: Agencia Regional de Desarrollo Productivo. 
Cuadro 4. Características de la provisión FIC.

\begin{tabular}{|l|l|}
\hline $\begin{array}{l}\text { Líneas } \\
\text { financiamiento, de } \\
\text { Resolución 277/2011 } \\
\text { de SUBDERE }\end{array}$ & $\begin{array}{l}\text { Investigación y desarrollo } \\
\text { Innovación en y para las empresas } \\
\text { Difusión y transferencia tecnológica } \\
\text { Aceleración del emprendimiento innovador } \\
\text { Formación, in serción y atracción de recursos } \\
\text { humanos especializados } \\
\text { Fortalecimiento de redes para la innovación y } \\
\text { equipamiento de apoyo a la competitividad } \\
\text { Fomento de la cultura del emprendimiento y la innovación } \\
\text { yel emprendimiento innovador }\end{array}$ \\
\hline $\begin{array}{l}\text { Líneas } \\
\text { financiamiento, de } \\
\text { Resolución O2/2016 } \\
\text { de SUBDERE }\end{array}$ & $\begin{array}{l}\text { La resolución } \mathrm{N}^{\circ} \text { 2 introdujo algunas modificaciones en las } \\
\text { líneas de financiamiento, quedando de la siguiente manera: } \\
\text { - Investigación (básica, aplicada, desarrollo } \\
\text { experimental, investigación y desarrollo). } \\
\text { - Innovación (empresarial, social, pública). } \\
\text { Difusión y transferencia tecnológica } \\
\text { - Aceleración del emprendimiento innovador. } \\
\text { - Formación, inserción y atracción de recursos } \\
\text { humanos especializados. } \\
\text { Fortalecimiento de redes para la innovación y } \\
\text { equipamiento de apoyo a la competitividad. } \\
\text { - Fomento de la cultura del emprendimiento y la } \\
\text { innovación, y el emprendimiento innovador. }\end{array}$ \\
\hline
\end{tabular}

Fuente: elaboración propia con base en SUBDERE, 2011 y SUBDERE, 2016.

\section{ANÁLISIS DE SU DESEMPEÑO}

\subsection{Distribución y asignación de los recursos}

En primer lugar, en lo que se refiere a la distribución de los recursos de la provisión entre los gobiernos regionales, entre los años 2008 y 2016 se han dispuesto M\$336.573.185 (cifras a precios constantes con base año 2008), los cuales se han distribuido siguiendo el algoritmo descrito en el punto anterior, de acuerdo a como se presenta en la Figura 2. 
Figura 2. Evolución de la distribución regional de la provisión FIC, años 2008, 2012 y 2016 (porcentajes).

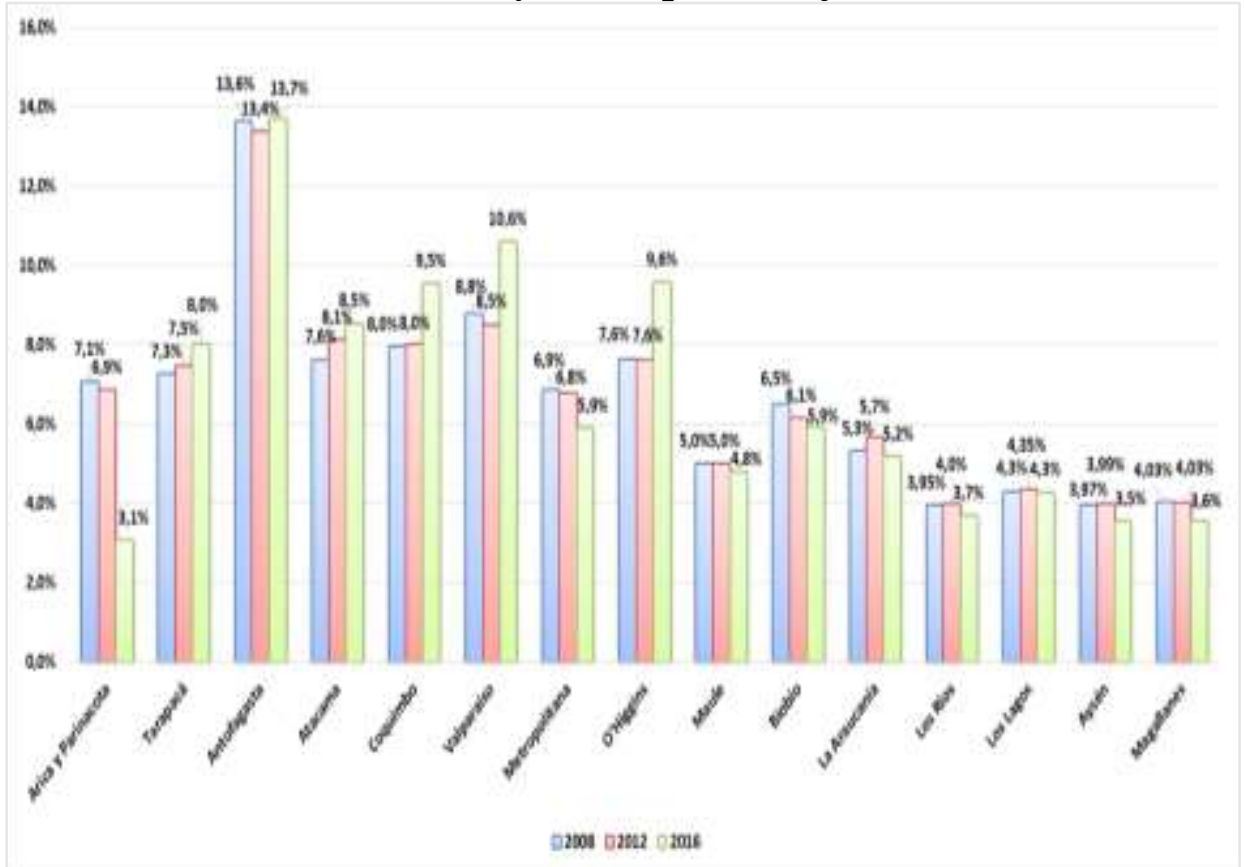

Fuente: elaboración propia con base en estadísticas de SUBDERE (años escogidos aleatoriamente para efectos de este trabajo).

Tal como se aprecia en el gráfico anterior, las mayores participaciones las han tenido las regiones de Antofagasta, Valparaíso y Coquimbo, mientras que las menores están en las regiones de Aysén, Magallanes y Los Ríos (el detalle de la distribución se entrega en el Anexo A). A continuación se recogen los promedios de participación regional en el periodo 2008-2016 en el Cuadro 5. 
Cuadro 5. Promedio de participación regional en la distribución de los recursos de la provisión FIC, periodo 2008-2016.

\begin{tabular}{|l|r|}
\hline Regiones & Promedio participación \\
\hline Antofagasta & $13,60 \%$ \\
\hline Valparaíso & $9,40 \%$ \\
\hline Coquimbo & $8,80 \%$ \\
\hline O'Higgins & $8,50 \%$ \\
\hline Atacama & $8,30 \%$ \\
\hline Tarapacá & $7,90 \%$ \\
\hline Metropolitana & $6,20 \%$ \\
\hline Biobío & $6,10 \%$ \\
\hline La Araucanía & $5,20 \%$ \\
\hline Arica y Parinacota & $5,10 \%$ \\
\hline Maule & $5,00 \%$ \\
\hline Los Lagos & $4,20 \%$ \\
\hline Aysén & $4,10 \%$ \\
\hline Los Ríos & $3,80 \%$ \\
\hline Magallanes & $3,80 \%$ \\
\hline Total & $100 \%$ \\
\hline
\end{tabular}

Fuente: elaboración propia con base en estadísticas de SUBDERE.

Los datos que se presentan a continuación fueron recopilados por SUBDERE desde los gobiernos regionales y corresponden a la asignación que cada uno de ellos realizó de los recursos del FIC-R que dispuso entre los años 2008 y 2016. Esto tiene como propósito verificar, entre otras cosas, el grado de uso que han tenido los recursos en relación a los fines que persigue y, como contrapartida, los recursos que quedan sin utilizarse o que son utilizados para otro tipo de iniciativas.

$\mathrm{Al}$ respecto, es importante considerar que no todos los gobiernos regionales disponen de registros completos, por lo que la información que será analizada presenta algunos vacíos.

\subsection{Análisis de la asignación de los recursos FIC-R por ejecutor}

En relación a su distribución por institución receptora, en el periodo 20082016, la mayor parte de los recursos fue entregada a entidades receptoras (entendiendo como tal a universidades, centros e institutos de investigación que cumplen con los requisitos establecidos en el reglamento que regula el funcionamiento de esta fuente de financiamiento), con un 52,5\%. Luego, están las agencias ejecutoras (que incluye a la Comisión Nacional de Investigación Científica y Tecnológica (CONICYT), la Corporación del Fomento de la Producción (CORFO), el Comité Innova Chile de CORFO, el Comité Innova Biobío de CORFO 6 , el Servicio de Cooperación Técnica (SERCOTEC), la Dirección de Promoción de Exportaciones

\footnotetext{
${ }^{6}$ Actualmente, este organismo forma parte del Comité de Desarrollo Productivo del Biobío el que, a su vez, integra el pilotaje que se realizó en materia de traspaso de competencias a los gobiernos regionales sobre fomento productivo e innovación entre los años 2015 y 2018.
} 
(ProChile), el Consejo Nacional de Producción Limpia (CPL), los Comité de Desarrollo Productivo Regionales (CDPR), y el Programa Iniciativa Científica Milenio (ICM), con un 44,7\%.

Entre ambos tipos de ejecutores abordan la mayor parte de los recursos. En ese sentido, es necesario considerar que los recursos que se entregan a agencias ejecutoras no son utilizados por ellas directamente, sino que los entregan a través de concursos a instituciones que forman parte de las entidades receptoras señaladas por lo que, en ese sentido, cumplen un rol de intermediario entre los gobiernos regionales y este tipo de organismos, disponiendo para ello su experiencia y capacidades en materia de formulación de instrumentos, evaluación de proyectos y el seguimiento y control de ellos. Esto lo financian con cargo a los gastos administrativos que se extraen de los recursos que se les asignan, los que oscilan entorno al 5\% del total asignado.

Además, se puede destacar la baja participación que tienen las corporaciones regionales de desarrollo productivo (CRDP), siendo probablemente uno de los motivos de ello las exigencias que establece la Ley Orgánica Constitucional sobre Gobierno y Administración Regional $\left(\mathrm{N}^{\circ} 19.175\right)$ la que, en su artículo 101 señala que los programas y/o proyectos que ejecuten solo podrán ser financiados hasta en un $50 \%$ con recursos de los gobiernos regionales. Lo expuesto se muestra en la Figura 3.

Figura 3. Total de recursos del FIC-R asignados por institución receptora, periodo 2008-2016 (porcentajes).

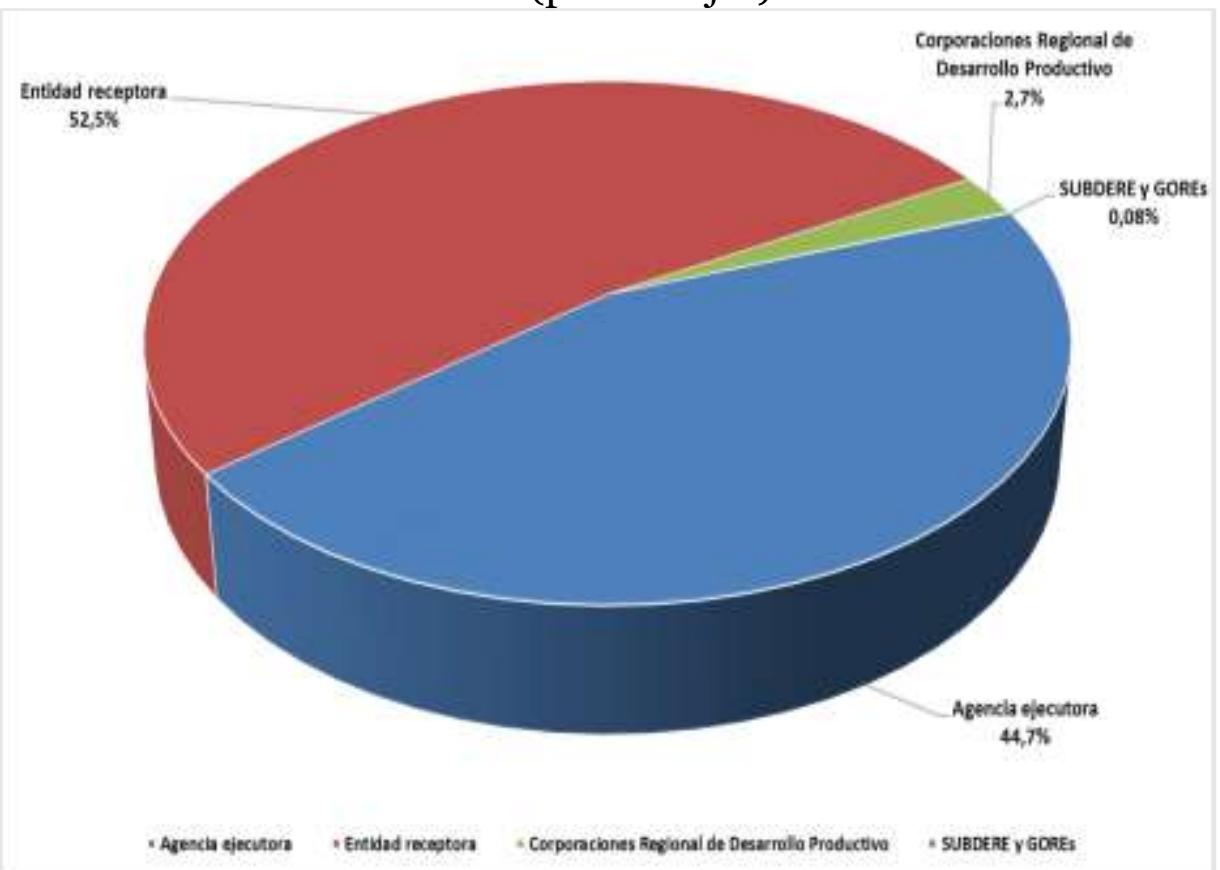

Fuente: elaboración propia a partir de datos provistos por los gobiernos regionales el año 2017.

En cuanto a la evaluación que ha tenido esta asignación, en igual periodo, la Figura 4 muestra principalmente el aumento que ha tenido la participación de las entidades receptoras y, como contrapartida, la disminución que ha tenido la 
participación de las agencias ejecutoras en los últimos años. Uno de los motivos que puede estar asociado a esto es reducción de las restricciones que progresivamente ha tenido la asignación del FIC, el que en los primeros años establecía porcentajes mínimos que debían ser asignados a agencias ejecutoras.

Figura 4. Asignación del FIC-R por institución receptora, periodo 2008-2016 (cifras en miles de pesos base 2008).

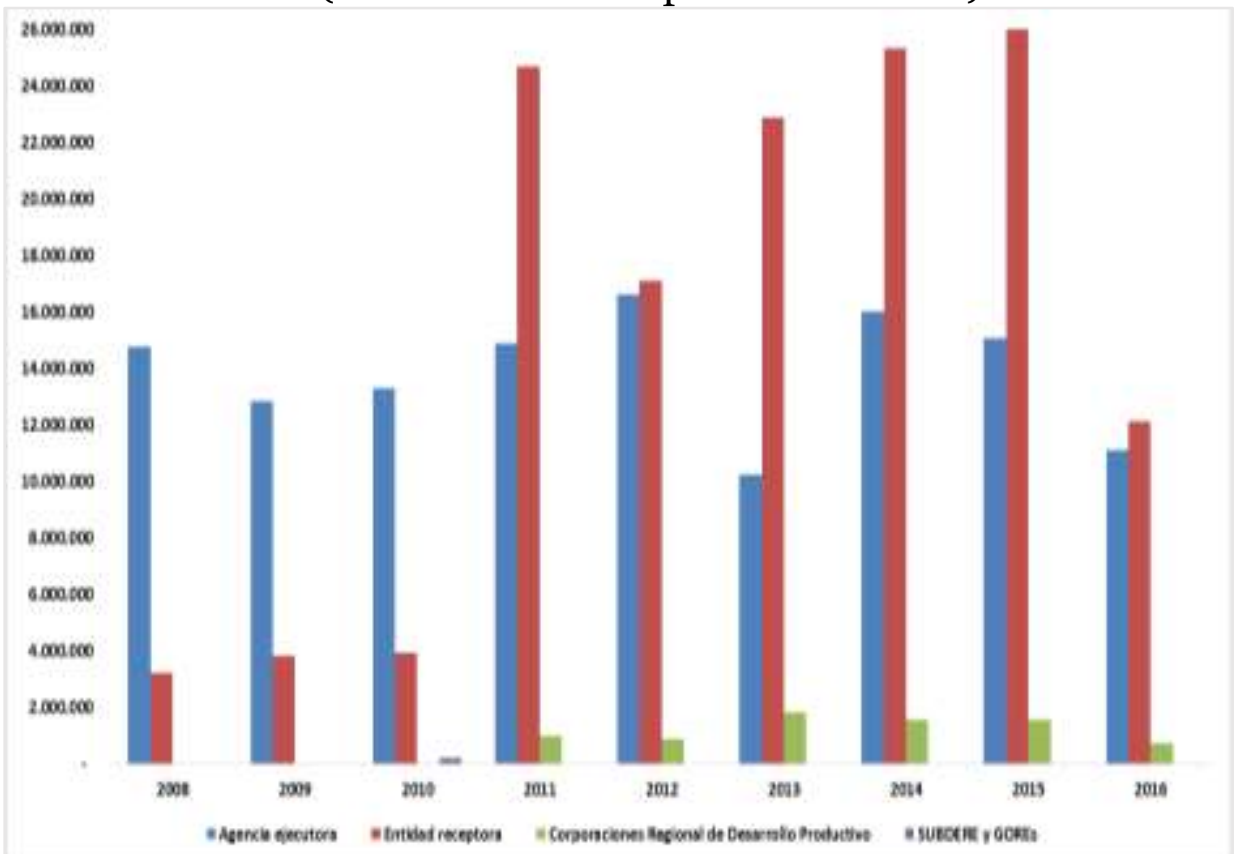

Fuente: elaboración propia a partir de datos provistos por los gobiernos regionales el año 2017.

En cuanto a su asignación por agencia ejecutora, los resultados en el periodo analizado se presentan en la Figura 5. En él se puede apreciar que, entre los años 2008 y 2016, CONICYT y CORFO han recibido la mayor parte de los recursos que se le han asignado a este tipo de institución receptora, representando entre las 2 más de un 50\% del total de los recursos entregados a agencias ejecutoras. Si se le suma a lo anterior al Comité Innova Chile de CORFO, que tiene un porcentaje de participación algo menor al de los organismos ya señalados, el porcentaje indicado llega a un $75 \%$. 
Figura 5. Total de recursos FIC-R asignados por agencia ejecutora, periodo 20082016 (porcentajes).

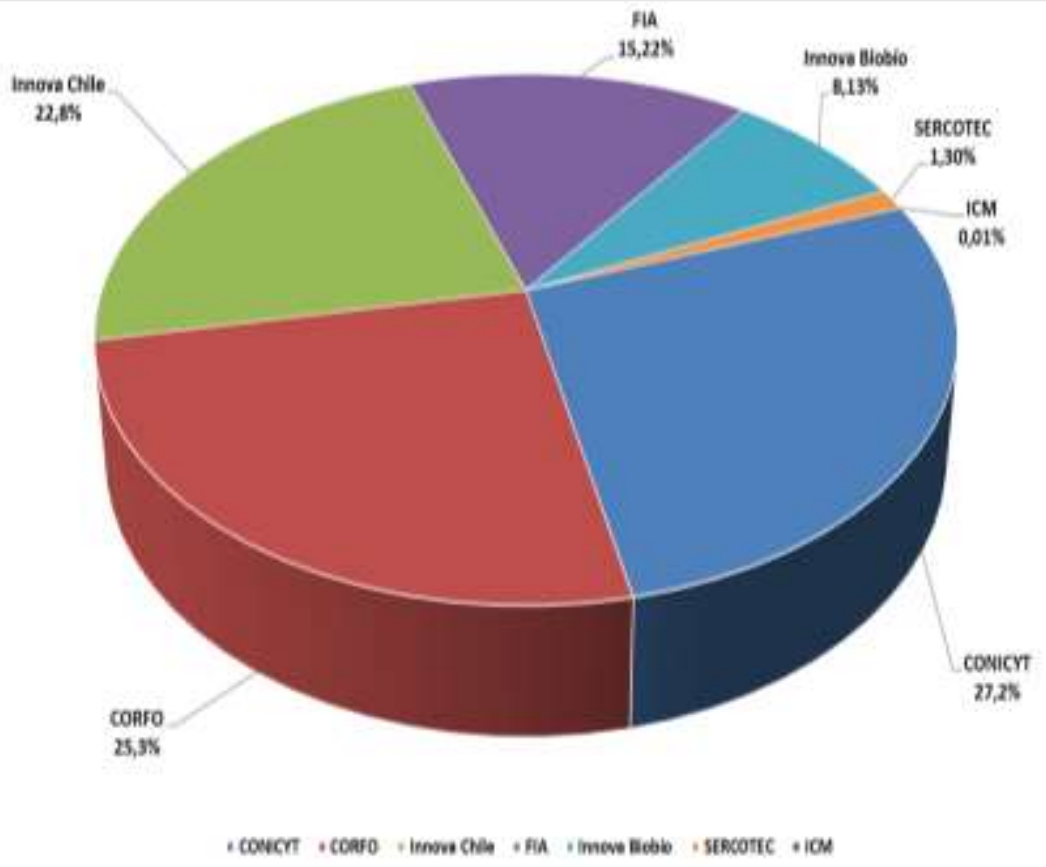

Fuente: elaboración propia a partir de datos provistos por los gobiernos regionales.

Luego, aparece la Fundación para la Innovación Agraria (FIA), la que tiene una participación de alrededor de un $15 \%$ dentro de las agencias ejecutoras.

Otro dato que es de interés es que no todas las agencias habilitadas para recibir recursos del FIC han aprovechado de manera relevante esta posibilidad (en relación a las agencias anteriormente señaladas), tal como ocurre con el caso de la ICM.

En lo que se refiere a su variación dentro del periodo en análisis, la Figura 6 destaca la baja que ha tenido la participación del Comité Innova Chile y CONICYT en los últimos años, así como también el relativo aumento que ha tenido la participación de CORFO y, enmenormedida, la Fundación para la Innovación Agraria. 
Figura 6. Asignación del FIC-R por agencia ejecutora, periodo 2008-2016 (cifras en miles de pesos base 2008).

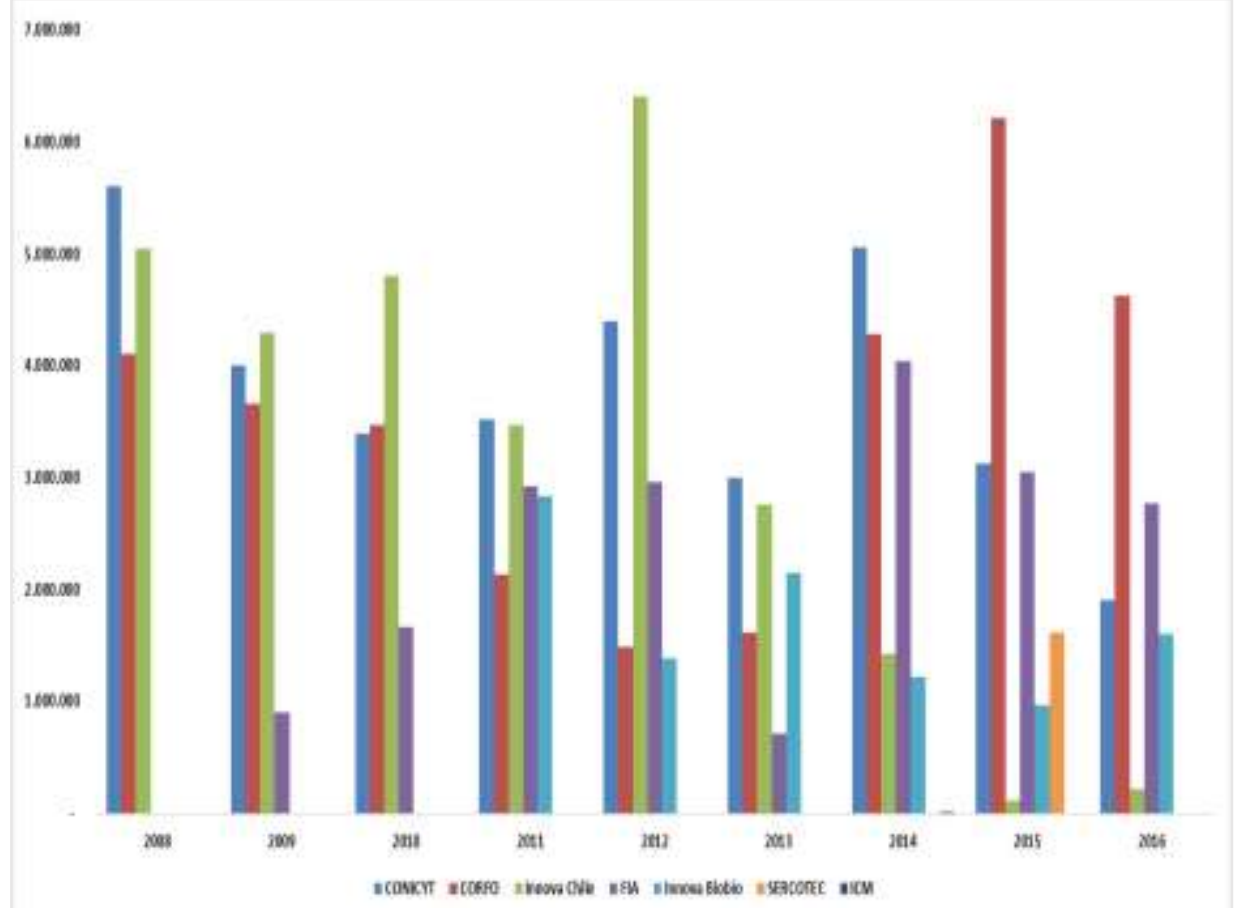

Fuente: elaboración propia a partir de datos provistos porlosgobiernos regionales el año 2017.

En lo que se refiere a la asignación realizada a entidades ejecutoras, la Figura 7 refleja una importante participación de las universidades dentro del monto total entregado a este tipo de instituciones receptoras, representando por sí solas 3/4 del total.

Figura 7. Total de recursos del FIC-R asignados por tipo de entidad receptora, periodo 2008-2016 (porcentajes).

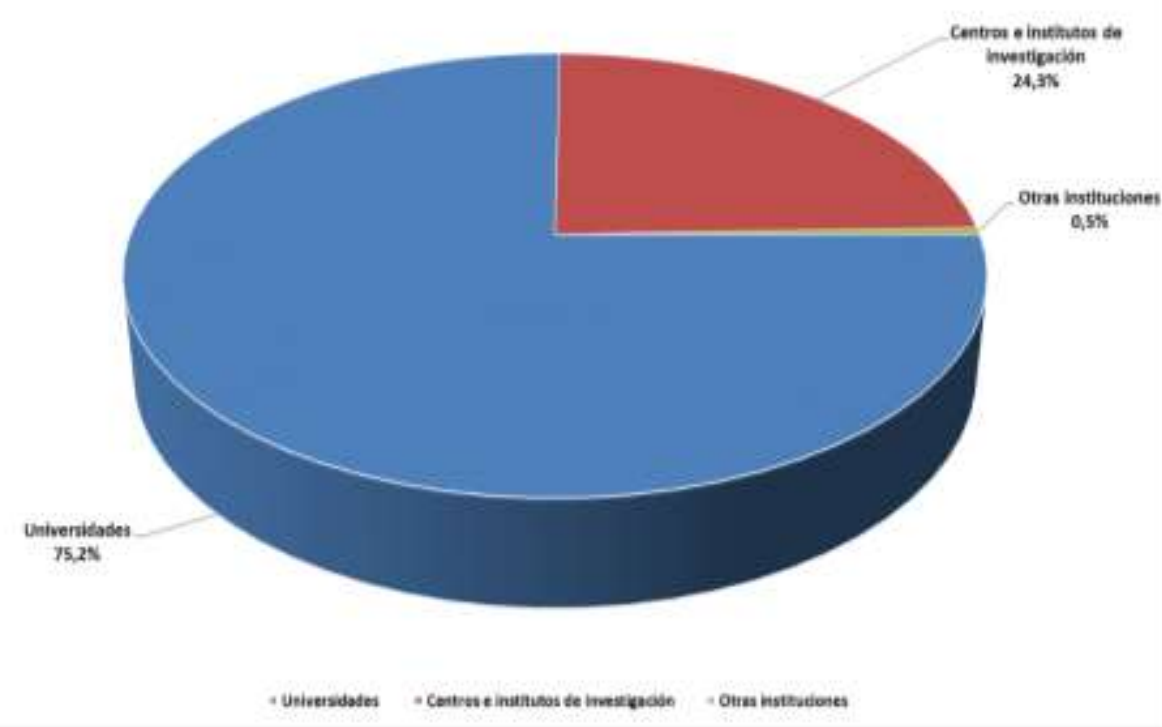

Fuente: elaboraciónpropia a partir de datosprovistos por los gobiernos regionales el año 2017. 
Luego, con una participación que cubre casi el cuarto restante, están los centros e institutos de investigación, entre los cuales se incluye a organismos que han nacido bajo el alero de universidades (tales como algunos centros regionales de desarrollo científico y tecnológico creados por el Programa Regional de CONICYT), y a organismos públicos de investigación, entre los que destaca el Instituto de Investigaciones Agropecuarias (INIA).

Dentro de las "otras instituciones" se incluye a empresas de servicios tecnológicos, tales como "Agua Marina Biominería S.A.”, entre otras similares.

La Figura 8 refleja la forma en que se ha dado esta asignación dentro del periodo analizado. En ese sentido, es destacable el importante aumento que ha tenido la participación de las universidades, lo que con fuerza se puede constatar desde el año 2011. Esto podría estar asociado a lo que se señaló anteriormente en relación a la reducción de las restricciones de porcentajes de asignación por tipo de institución receptora.

Figura 8. Asignación del FIC-R por tipo de entidad receptora, periodo 2008-2016 (cifras en miles de pesos base 2008).

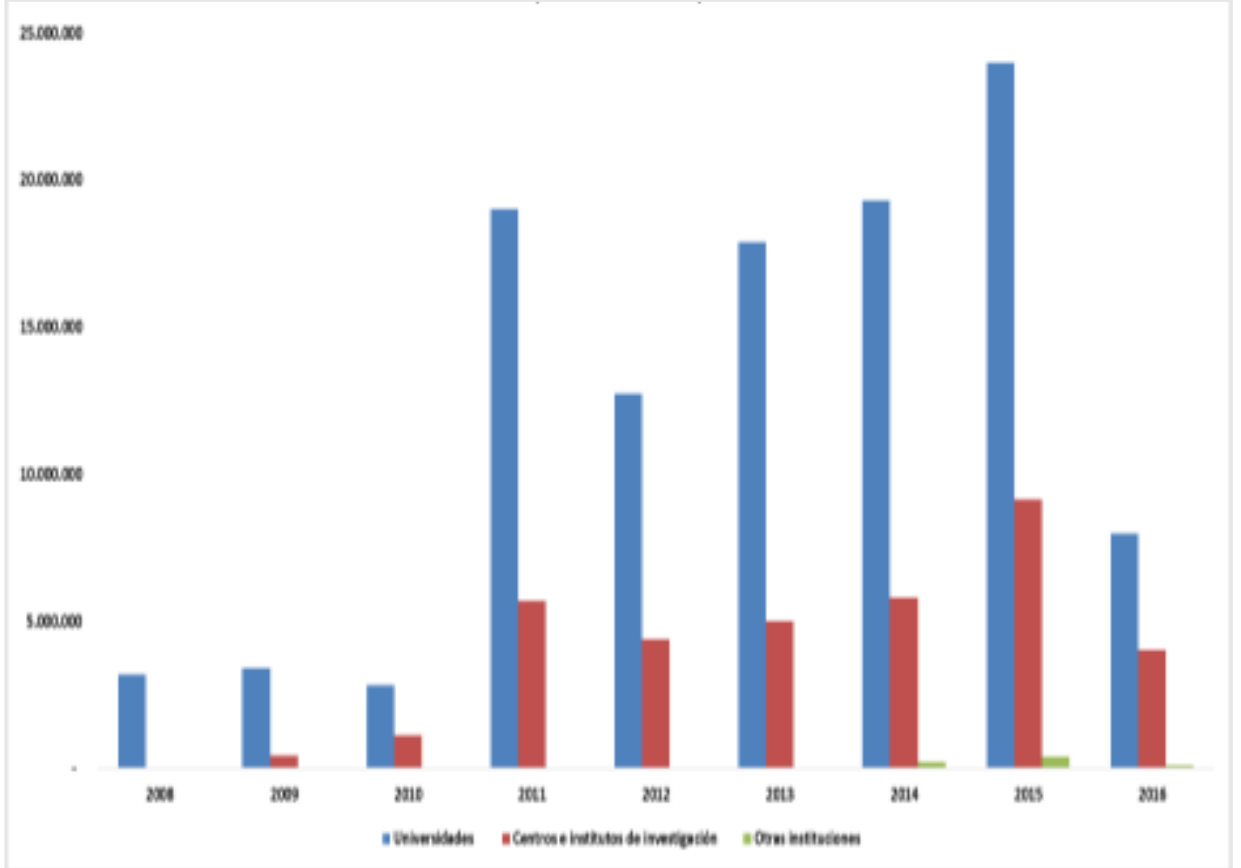

Fuente: elaboración propia a partir de datos provistos por los gobiernos regionales.

Por su parte, los centros e institutos de investigación también han tenido en su participación, principalmente desde el año 2012, aunque en una proporción menor respecto a la lograda por las universidades.

En relación a lo anterior, son diversas las universidades que han participado en la asignación de recursos del FIC, tal como lo muestra el cuadro $\mathrm{N}^{\circ} 8$. Sobre esto, es importante considerar que su participación en la asignación no necesariamente se circunscribe a la o las regiones donde tienen sedes, sino que ha trascendido a varias regiones. Esto puede explicar la participación destacada no solo de las llamadas universidades nacionales, tales como la Universidad de Chile y la Pontificia 
Universidad Católica de Chile, sino que también de universidades regionales como las que se presentan en el Cuadro 5 .

Cuadro 5. Participación de las universidades e institutos profesionales en la asignación del FIC, en el periodo 2008-2016.

\begin{tabular}{|l|r|}
\hline Universidades e institutos profesionales & Porcentaje del total \\
\hline Universidad Católica del Norte & $8,3 \%$ \\
\hline Universidad de Antofagasta & $7,9 \%$ \\
\hline Universidad Austral & $7,8 \%$ \\
\hline Universidad Arturo Prat & $5,1 \%$ \\
\hline Universidad de Chile & $4,9 \%$ \\
\hline Universidad de Los Lagos & $4,6 \%$ \\
\hline Universidad Católica de Chile & $3,3 \%$ \\
\hline Universidad Santo Tomás & $3,2 \%$ \\
\hline Universidad Técnica Federico Santa María & $3,0 \%$ \\
\hline Universidad de Tarapacá & $3,0 \%$ \\
\hline Otras universidades & $48.9 \%$ \\
\hline Total & $100 \%$ \\
\hline
\end{tabular}

Fuente: elaboración propia a partir de datosprovistos por los gobiernos regionales el año 2017.

4.3. Percepción de actores relevantes: encuesta efectuada por SUBDERE en materia de percepción del funcionamiento del FIC-R

El objetivo de la encuesta fue obtener información que fuera de utilidad para evaluar el desempeño de esta provisión y plantear recomendaciones para su mejoramiento. Para eso, se formularon preguntas en los ámbitos de "Perfil y grado de conocimiento de la provisión en objetivos, funcionamiento, tipos de proyectos y resultados"; "Grado de pertinencia de las líneas financiamiento"; "Acciones de difusión, asignación y seguimiento"; "Actores"; "Relación con la ERI" y "Posibilidades de mejora".

La muestra, no probabilística, incluye respuestas enviadas por:

- Funcionarios de los gobiernos regionales (GORE).

- Funcionarios agencia.

- Entidades Ejecutoras.

- Consejeros Regionales (CORE).

- Beneficiarios (información entregada por GORES).

El cuestionario fue aplicado en línea entre los días 14 y 28 de marzo de 2017. Respondieron 310 personas, las que se distribuyen manera descrita en la Figura $9^{7}$.

7 En el caso de los Beneficiarios, cabe tener en cuenta que gran parte de los contactos provienen de la región de O'Higgins. Sobre los CORE, solo se recibieron 6 respuestas, por lo que se analizan aparte. 
Figura 9. Distribución de encuestas respondidas por perfil (porcentajes).

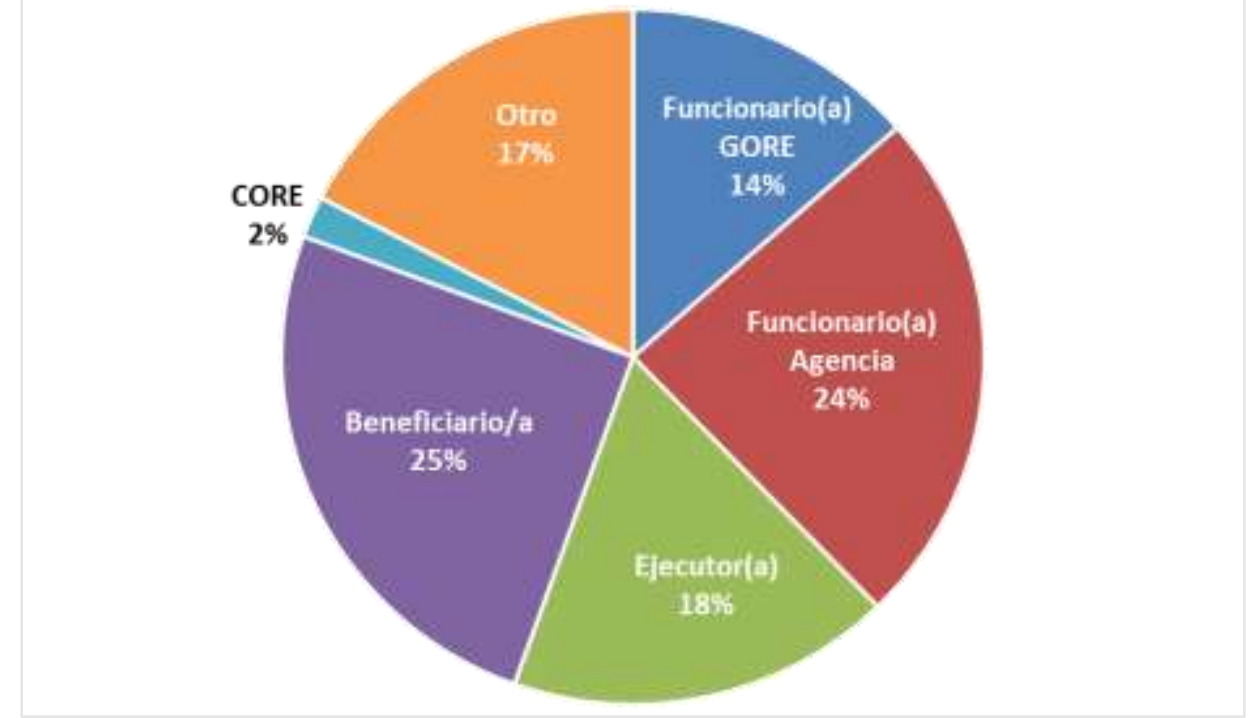

Fuente: elaboración propia.

En general, la mayoría de ellos declara conocer bien o muy bien distintos aspectos de la provisión (objetivos, tipos proyectos y funcionamiento), mientras que se conoce poco sobre la articulación entre actores y los resultados.

Por su parte, en relación a la valoración de pertinencia según líneas de financiamiento, se analiza la valoración de la pertinencia con el interés regional, de las siguientes líneas de financiamiento:

- Investigación básica.

- Investigación aplicada.

- Desarrollo experimental.

- Investigación y desarrollo.

- Innovación empresarial.

- Innovación pública.

- Innovación social.

- Difusión y transferencia tecnológica.

- Aceleración del emprendimiento innovador.

- Formación, atracción e inserción de recursos humanos especializados.

- Fortalecimiento de redes para la innovación.

- Equipamiento de apoyo para la competitividad.

- Fomento de la cultura del emprendimiento e innovación, y el emprendimiento innovador.

$\mathrm{Al}$ respecto, la apreciación de cuán pertinentes al interés regional son cada línea de financiamiento, es más bien común y transversal a todos los perfiles encuestados. Si se considera solo aquellos que conocen bien los objetivos, o resultados o tipos de proyecto, las líneas consideradas más pertinentes son $\mathrm{I}+\mathrm{D}$, Innovación empresarial y Fortalecimiento de redes para la innovación. Por su parte, no resultan tan relevantes la Innovación pública, la innovación social y el desarrollo experimental.

Para los ejecutores y funcionarios de agencias, también resultan importantes la difusión y transferencia tecnológicayla aceleración del emprendimiento innovador. 
Llama la atención que, transversalmente, independiente del nivel de conocimiento de la provisión, se consideran que la investigación básica no es pertinente con el interés regional.

En lo que se refiere a la percepción que existe sobre los procesos de difusión, asignación y seguimiento de la ejecución de los recursos de la provisión, se extrajo lo siguiente:

- Difusión: En general, no hay acuerdo sobre la calidad de la difusión, si se considera solo a aquellos que conocen el funcionamiento de la provisión (46\% le parece adecuada, mientras que para el $42 \%$ es inadecuada).

- Si se analiza por perfil, se encuentra que para los funcionarios de los GORE, se repite el patrón. Sin embargo, para los funcionarios de agencias, solo un $28 \%$ la considera adecuada, mientras que $59 \%$ no la considera adecuada.

- Para los ejecutores y beneficiarios, están de acuerdo con que la difusión es adecuada.

- Asignación: A nivel global, no existe acuerdo sobre la calidad del proceso de asignación de los recursos.

- Si se considera solo a aquellos que declaran conocer el funcionamiento de la provisión, recién un 50\% considera que es adecuada. Sin embargo, aún un $38 \%$ está en desacuerdo.

- Analizando los datos por cada perfil, entre los funcionarios de los GORE no existe diferencia; para los funcionarios de las Agencias, predomina una mala valoración de la asignación mientras que, por el contrario, para los ejecutores, predomina una buena valoración. Por su parte, para los Beneficiarios tampoco existe diferencia. En general no se observa marcadamente una buena apreciación de la asignación de los concursos y de la provisión.

- Seguimiento: Al igual que con la asignación, a nivel global, no predomina una apreciación sobre el proceso de seguimiento del uso de los recursos. Ahora bien, si se considera solo aquellos que conocen del funcionamiento, hay una mejor valoración del proceso de seguimiento ( $57,8 \%$ lo considera adecuado).

- Respecto a la posición por cada perfil, para los Funcionarios de los GORE, nuevamente no existe diferencia; para los funcionarios de las Agencias y los beneficiarios, se observa una buena valoración del seguimiento (mayor a $50 \%)$, mientras que, entre los Ejecutores y los Beneficiarios, predomina una muy buena valoración (72\%). En general hay concordancia en que el seguimiento es adecuado, curiosamente esto no sucede en los funcionarios de los GORE (ni para los CORE).

En síntesis, en estas tres etapas de ejecución de la provisión, para los funcionarios de los GORE, no se ve una concordancia en si son llevadas adecuadamente o no. Además, los ejecutores en general tienen una buena valoración de la forma en que se realizan estos tres procedimientos. No existe ningún patrón claro en cuanto a la valoración del proceso de asignación, siendo el seguimiento la única etapa donde se puede apreciar consenso, en cuanto a que es bien llevado.

En otro ámbito, sobre los actores que participan del funcionamiento del FIC-R, en general y de forma transversal al perfil y grado de conocimiento en los distintos aspectos del funcionamiento de la provisión, se constata que cada actor es relevante en los roles que se espera lo sea, por ejemplo: 
- Ministerio de Economía, Fomento y Turismo (MINECON), Consejo Nacional de Innovación para el Desarrollo (CNID), SUBDERE, los CORE, la Sociedad Civil y las Asociaciones Gremiales, son considerados relevante para la definición de lineamientos.

- MINECON, Dirección de Presupuestos (DIPRES) y los CORE también son relevantes para la asignación de recursos.

- Los Centros de investigación y las Empresas son relevantes principalmente para la ejecución. Lo mismo con las Agencias, pero también tienen importancia relativa en la asignación de recursos.

- A las Divisiones de los GORE se les asigna principalmente un rol en el seguimiento y evaluación, y no tanto en la definición de lineamientos.

- SUBDERE tiene participación relativa en distintas etapas, entre ellas asignación de recursos, definición de lineamientos y seguimiento.

En cuanto a su articulación entre los actores, en general y transversalmente a los perfiles encuestados, es en su mayoría considerada buena, sin embargo, ni por perfil ni en general, dicho porcentaje supera el $55 \%$ de las apreciaciones. Solo desde el punto de vista de encuestados que son parte de entidades ejecutoras, se considera mayoritariamente que existe una buena articulación entre actores.

En relación a las Estrategias Regionales de Innovación (ERI) y, en general, a los instrumentos de planificación regional en estos temas, a nivel global, solo es conocida por el $48 \%$ de los encuestados, mientras que un 30\% de ellos declara no conocerla, como muestra la Figura 10. El grupo que mayoritariamente conoce la ERI son funcionarios GORE. No obstante, de todas maneras, se considera importante en general para orientar la innovación regional a través de instrumentos como las ERI.

Figura 10. Grado de conocimiento de la ERI, por perfil (como porcentaje por perfil de actor).

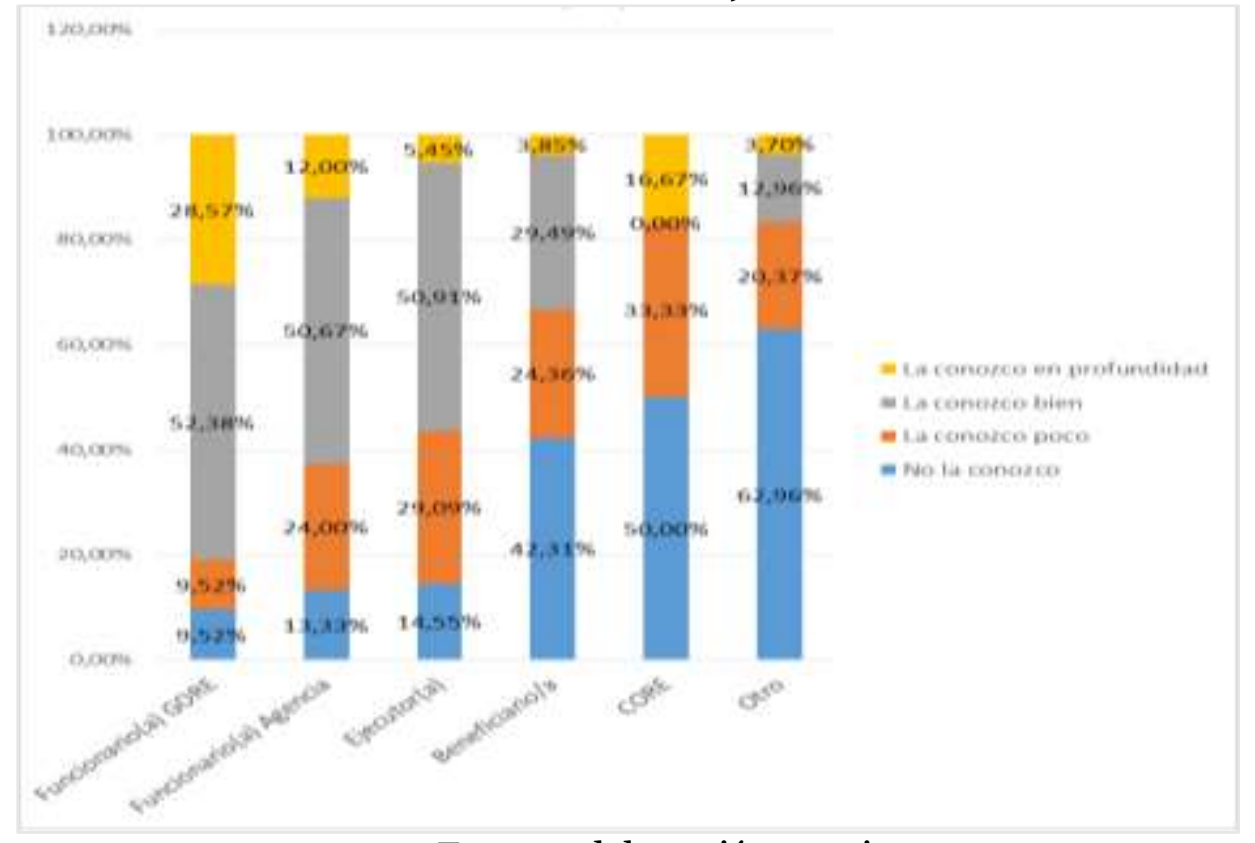

Fuente: elaboración propia. 
Por último, se sometieron a consideración de los encuestados, las siguientes propuestas de mejora:

- Incluir mecanismos de evaluación de resultados e impacto.

- Mecanismos formales que permitan mejorar pertinencia instrumentos de las Agencias, con el interés regional.

- Contar con instancias técnicas regionales para evaluar los proyectos postulados y sus ejecutores.

- Diferenciar líneas de acción de corto, mediano y largo plazo.

- Potenciar cofinanciamiento público-privado.

- Definición por parte de cada GORE de su abanico de ejecutores.

- Modificar criterios de distribución de recursos entre regiones.

- Destinar parte de los recursos a implementar ERI.

El grado de valoración por parte de los encuestados sedescribe en el Cuadro 6.

Cuadro 6. Valoración de propuestas de mejora para la provisión FIC.

\begin{tabular}{|l|r|l|}
\hline Propuesta mecanismos de & Porcentaje & Consideraciones \\
\hline $\begin{array}{l}\text { Incluir } \\
\text { evaluación de resultados e } \\
\text { impacto }\end{array}$ & $78 \%$ & $\begin{array}{l}\text { Priorizado por aquellos que } \\
\text { conocen los objetivos y los } \\
\text { resultados de la provisión }\end{array}$ \\
\hline $\begin{array}{l}\text { Mecanismos formales que } \\
\text { permitan mejorar pertinencia } \\
\text { instrumentos las Agencias, } \\
\text { con el interés regional }\end{array}$ & $68 \%$ & \\
\hline $\begin{array}{l}\text { Contar con instancias técnicas } \\
\text { regionales para evaluar los } \\
\text { proyectos postulados y sus } \\
\text { ejecutores }\end{array}$ & $62 \%$ & $\begin{array}{l}\text { Priorizado por conocimiento } \\
\text { resultados, objetivos y } \\
\text { funcionamiento }\end{array}$ \\
\hline $\begin{array}{l}\text { Diferenciar líneas de acción de } \\
\text { corto, mediano y largo plazo }\end{array}$ & $60 \%$ & $\begin{array}{l}\text { Priorizado por conocimiento de } \\
\text { objetivos y funcionamiento }\end{array}$ \\
\hline $\begin{array}{l}\text { Potenciar cofinanciamiento } \\
\text { público-privado }\end{array}$ & $49 \%$ & $\begin{array}{l}\text { Priorizado por conocimiento de } \\
\text { objetivos y funcionamiento }\end{array}$ \\
\hline $\begin{array}{l}\text { Definición por parte de cada } \\
\text { GORE de su abanico de } \\
\text { ejecutores }\end{array}$ & $45 \%$ & $\begin{array}{l}\text { Para los funcionarios GORE esta } \\
\text { propuesta es prioritaria (más } \\
70 \% \text { aprobación) }\end{array}$ \\
\hline $\begin{array}{l}\text { Modificar criterios de } \\
\text { distribución de recursos entre } \\
\text { regiones }\end{array}$ & $44 \%$ & \\
\hline $\begin{array}{l}\text { Destinar parte de los recursos a } \\
\text { implementar ERI }\end{array}$ & $35 \%$ & Excepto gobiernos regionales \\
\hline
\end{tabular}

Fuente: elaboración propia.

4.4. Conclusiones extraídas del taller de trabajo realizado el 29 de agosto de 2017

El taller denominado "Análisis del estado actual y temas por abordar del Fondo de Innovación para la Competitividad de asignación regional", fue realizado el 29 de agosto de 2017, en dependencias de la SUBDERE. 
Asistieron representantes de las siguientes instituciones, todas ligadas al proceso de operación de él:

- Agencia de Sustentabilidad y Cambio Climático (ex CPL).

- Agrupación de Universidades Regionales, AUR.

- Consejo Nacional de Innovación para el Desarrollo, CNID.

- Consejo Regional de Antofagasta.

- Comisión Nacional de Investigación Científica y Tecnológica, CONICYT.

- Fundación para la Innovación Agraria, FIA.

- Ministerio de Economía, Fomento y Turismo, MINECON.

- Subsecretaría de Desarrollo Regional y Administrativo, SUBDERE.

- Servicio de Cooperación Técnica, SERCOTEC.

Además, en calidad de beneficiarios, fueron invitados representantes de la Universidad Central y del Centro Regional de Estudios en Alimentos Saludables, CREAS, de la región de Valparaíso.

A continuación se presentan las principales ideas planteadas en la actividad:

- Sobre Lineamientos asociados a las decisiones del FIC-R.

- Se sugiere incorporar en el financiamiento la contratación de expertos para la evaluación técnica de ciertos proyectos que lo requieren, ya que los gobiernos regionales no disponen de los recursos para ello.

- Alta valoración del FIC y la Estrategia Regional de Desarrollo (ERI) dentro de los actores regionales ligados al tema. Esto se ha expresado en que más del 90\% de la asignación del FIC se ha asignado a partir de la ERI.

- Relacionado con lo anterior, se destaca la importancia de contar con instrumentos de planificación ligados al tema.

- Es necesario evaluar los resultados obtenidos a partir de las ERI.

- Se destaca que el FIC ha servido para que el GORE reemplace la inversión que realizaba con recursos del presupuesto regular en materia de ciencia, tecnología e innovación. Además, el FIC ha sido utilizado para compensar la falta de inversión sectorial en temas de innovación, ciencia y tecnología, en algunas regiones.

- El FIC les brinda la oportunidad a las regiones de diversificarse desde el punto de vista de su matriz productiva.

- Foco estratégico en la ejecución del FIC-R y principales socios.

- Se requiere mejorar la evaluación (especialmente expost) y monitoreo de la ejecución del FIC.

- Es necesario promover el trabajo en red para el desarrollo de proyectos conjuntos, así como también el trabajo interregional en temas de interés común.

- De parte de las agencias ejecutoras, se requiere que se coordinen entre sí y que propongan instrumentos innovadores acordes a las necesidades de las regiones.

- En cuanto a la operación del FIC, se destaca la alta rotación de equipos dentro de los gobiernos regionales. También se destaca el sobre compromiso que existe de los fondos.

Entre las propuestas están las siguientes:

- Prohibir la disminución del FIC dentro del presupuesto regional. 
- Establecer normas gráficas generales que permitan posicionar el fondo.

- Eliminar la visación de DIPRES de las resoluciones de modificación presupuestaria (que, muchas veces, retrasan la entrega de recursos), o disminuir su tiempo de tramitación.

- Incluir a cooperativas y asociaciones gremiales dentro de los ejecutores.

- Incorporar a expertos dentro de los procesos de evaluación y seguimiento de los proyectos.

- Incorporar a municipalidades. En este ámbito, algunos gobiernos regionales ya ha desarrollado algunas experiencias en temas transversales y de turismo.

- Aspectos obstaculizadores en la operación y en la evolución del FIC-R y propuestas de mejoras.

- Se hace hincapié en los extensos tiempos de tramitación (3 meses), los cuales retrasan la ejecución del presupuesto.

- También se señala la atomización de proyectos y la falta de interrelación y continuidad entre ellos.

- Se reiteran los problemas en el ámbito presupuestario por cuestiones de carácter administrativo.

En lo referido a recomendaciones:

- El seguimiento en terreno de los proyectos, sustentabilidad, aplicación de resultados. Así también la integración, integralidad y adecuación de los proyectos, por último, se menciona la adaptación de los instrumentos de financiamiento.

- Se debería permitir a la región poner condiciones especiales. El Gobierno Central debe actuar como articulador, no trabar los procesos.

- Fortalecer procesos mineros sustentables.

- Incorporar en la ley de presupuestos la prohibición de disminuir el presupuesto del FIC.

- Fortalecer la gobernanza y la articulación de actores.

A partir de los análisis efectuados en este punto y en los anteriores, se ha logrado recopilar diversos aspectos estratégicos y operativos relacionados con el funcionamiento de la provisión FIC-R los que, a su vez, se refieren a temas tales como el cumplimiento de su objetivo de política pública y los efectos que se derivan de la normativa que rige su operación (reglamentos), tanto en lo que se refiere a su proceso de distribución y asignación como de ejecución y evaluación, abordando los alcances de los roles que asumen los diversos actores que están ligados a ellos.

Esto permite deducir y extraer una serie de ideas en relación al desempeño de esta fuente regional de financiamiento que permiten sustentar las conclusiones y recomendaciones que se presentan a continuación. En términos generales, los planteamientos que se hacen apuntan precisamente a mejorar algunas definiciones de objetivos y lineamientos del FIC-R, así como también su operación, de tal manera de que permita abordar sus principales debilidades y potenciar la utilidad del instrumento para que los gobiernos regionales hagan frente a los desafíos actuales y futuros que presenta la competitividad de sus territorios. 


\section{CONCLUSIONES}

A partir de los análisis realizados en los puntos anteriores, se pueden plantear las siguientes conclusiones:

- Algunos focos actuales que plantea la literatura dicen relación con la promoción de especialización inteligente, con la aceleración de procesos de innovación mediante la articulación entre entidades ubicadas en distintas partes del mundo y con la deslocalización de actividades productivas, entre otras tantas. En ese sentido, se hace necesario pensar en intervenciones públicas más audaces desde el punto de vista de los efectos que se busca obtener y que sean detonadoras de inversión privada y extranjera que genere sinergias y círculos virtuosos de ampliación y mayor complejización de los sectores productivos (basadas en las capacidades actuales y potenciales de cada región) y de mejoramiento de la productividad.

- Los cambios que se han producido en la provisión, que en general se han traducido en una mayor descentralización y empoderamiento de los gobiernos regionales con el uso de los recursos que se les entregan año a año, no han ido de la mano con un acompañamiento desde el nivel central, lo cual se ha traducido en un cierto vacío institucional que ha provocado algunas situaciones no deseables, tales como la falta de información sistematizada y procesada respecto al desempeño de esta fuente de financiamiento en cada región. Esto trae consigo la necesidad de volver a establecer una organización y roles entre entidades del nivel central con foco en apoyar y articular actores, tal como se propone más adelante.

- Relacionado con lo anterior, existe disparidad de niveles de información sobre el desempeño de la provisión en los distintos gobiernos regionales, lo cual no permite visualizar con claridad los resultados que han obtenido y contar con insumos que permitan justificar su importancia y existencia.

- Los cambios señalados (principalmente respecto a la eliminación de la obligación de asignar un porcentaje mínimo a agencias públicas) han modificado la participación de los organismos ejecutores en la asignación de recursos, observándose en general un aumento de la participación directa de las universidades y de los centros e institutos de investigación y una disminución de la participación de las agencias ejecutoras como CONICYT, CORFO y otras. Esta baja también es posible atribuirla a la poca diversificación de los instrumentos de financiamiento de las agencias, respecto a los lineamientos señalados en las estrategias Regionales de Innovación y las prioridades de los gobiernos regionales, como también los criterios de asignación de los recursos a los beneficiarios y los tiempos asociados a su ejecución. La introducción de nuevas agencias (tales como el Consejo Nacional de Producción Limpia (CPL) y el Servicio de Cooperación Técnica (SERCOTEC), entre otras) podría alterar nuevamente esta tendencia, en la medida en que su oferta resulta atractiva para los gobiernos regionales.

- Las nuevas líneas de financiamiento incorporadas recientemente (como innovación social e innovación pública) podrían ampliar el abanico de intervención del FIC regional hacia otras dimensiones de la competitividad reconocidas internacionalmente (como la de institucionalidad). Esto trae 
consigo la necesidad y, a la vez, oportunidad de generar o fortalecer las capacidades regionales en otras materias. No obstante, es importante considerar la necesidad de no dispersar en exceso los recursos, de tal forma de que no produzcan los efectos deseados, tales como que se financie una gran cantidad de iniciativas, pero de bajo impacto.

- Desde el punto de vista de la ejecución de los recursos, los tiempos asociados a la definición de las entidades ejecutoras, los mecanismos de identificación y asignación de los recursos, la suscripción de los convenios de transferencias, las facultades delegadas en las autoridades regionales para la firma de los convenios, las revisiones de las respectivas fiscalías, el cumplimiento de las disposiciones de establecidas en la Resolución $\mathrm{N}^{\circ} 30$ de la Contraloría General de la República sobre las rendiciones de transferencias, las modificaciones de contratos y los plazos de ejecución de los estudios, programas y proyectos, son razones esgrimidas por los gobiernos regionales para justificar la subejecución de los recursos de un año presupuestario.

\section{ReCOMENDACiONES}

En función de los planteamientos actuales que existen en materia de políticas de innovación y de desarrollo productivo e industrial, así como también respecto a los desafíos que presenta el desarrollo económico del país (especialmente en cuanto a la diversificación y complejización de su matriz productiva y al mejoramiento de la productividad) y los incentivos que genera el ciclo electoral en relación al tipo de inversiones a priorizar, se hace necesario estructurar el trabajo en materia de innovación como fuente para el mejoramiento de la competitividad con acciones de corto, mediano y largo plazo. Para ello, se sugiere evaluar la posibilidad de reestructurar el FIC Regional dividiéndolo en 2 componentes, los cuales pueden actuar en simultáneo o de manera focalizada en uno de ellos:

- Componente 1: para acción que logre resultados de corto y mediano plazo, basada en temas tales como difusión (catch-up) y transferencia tecnológica; desarrollo tecnológico; capacitación y perfeccionamiento de capital humano; emprendimiento innovador; generación y fortalecimiento de redes para la innovación y la adquisición y mejoramiento de equipamiento para el desarrollo de la innovación (como el utilizado en procesos de investigación científica y tecnológica, por ejemplo) y sus fuentes (como la ciencia y la tecnología); entre otros.

- Componente 2: para acción que permita generar y potenciar capacidades para obtener resultados de largo plazo, mediante la investigación científica básica y aplicada; atracción, inserción y formación de capital humano avanzado y generación de una cultura que permita el reconocimiento y la valoración de la innovación y la ciencia y tecnología desde temprana edad; entre otros.

- En ese sentido, es importante considerar que este tipo de proyectos logran sus resultados principales en un periodo largo de tiempo (más de tres años), lo cual hace necesario para promoverlos contar con instrumentos que permitan asegurar el financiamiento plurianual. Por esto, se recomienda promover el uso de instrumentos tales como convenios de programación u otros similares, que permitan también comprometer recursos de organismos del nivel central e, incluso, de privados. 
Para su operación se recomienda abordar temas como los siguientes:

- Definición de alcances de cada componente: a partir tanto de lineamientos establecidos a nivel nacional (planteados por organismos como el Consejo Nacional de Innovación para el Desarrollo - CNID), como por cada gobierno regional, con base en un instrumento de planificación regional (como estrategias o políticas regionales de innovación) que cada uno defina como vinculante para enmarcar la asignación de los recursos de la provisión.

- Definición de características mínimas que deben cumplir los proyectos financiables: se recomienda promover el cofinanciamiento de los proyectos de parte de sus responsables y beneficiarios, mediante su valoración positiva dentro del proceso de evaluación de proyectos (considerando para ello, a modo de referencia, indicadores de ciencia, tecnología, innovación y emprendimiento) $\mathrm{y} / \mathrm{u}$ otra forma que no desincentive en exceso la participación. Asimismo, se recomienda establecer plazos de ejecución (hasta 3 años para el componente 1 y sin plazo para el componente 2), montos máximos a solicitar y la obligación de establecer instancias de difusión y articulación con actores relevantes ligados al tema de cada proyecto, entre otras consideraciones.

- Aseguramiento de la pertinencia y de la calidad de los proyectos. Dado que resulta imprescindible asegurar la pertinencia de los proyectos en relación a los lineamientos del fondo y al interés regional y su calidad en esa perspectiva, se recomienda fortalecer el proceso de evaluación de proyectos mediante:

- La estructuración del proceso en una instancia regional, que evalúe la pertinencia del proyecto en relación a su interés (conformada por representantes de sectores público, privado y académico) y una instancia técnica, que los evalúe desde el punto de vista científico-técnico (conformada por expertos en los temas priorizados por cada gobierno regional para el uso de los recursos y que sean designados por cada intendente con acuerdo del consejo regional respectivo, los que pueden ser de otras regiones y/o extranjeros para reducir el riesgo de existencia de conflictos de interés; y financiada con recursos de la propia provisión), las cuales de manera secuencial definan una propuesta de proyectos a financiar, sobre la que deberán pronunciarse el Intendente y el Consejo Regional. Es fundamental establecer que esta última instancia no tendrán como rol evaluar los proyectos, sino que solamente pronunciarse respecto de la propuesta que se les presente, de tal manera de evitar influencias que no corresponden para el uso de este tipo de recursos.

- Se propone que las bases de las convocatorias que realicen los gobiernos regionales sean revisadas y visadas por las mismas instancias señaladas, para su aprobación por parte del intendente y del consejo regional respectivo.

Para efectuar el seguimiento de los proyectos, se recomienda utilizar las instancias propuestas en el punto anterior, las que serían apoyadas por una instancia operativa conformada por profesionales de las divisiones de planificación y desarrollo y análisis y control de gestión. Luego, una vez que entren en funcionamiento, se sugiere incorporar a profesionales de las divisiones de fomento e industria. 
En este contexto, sería de utilidad promover el aprovechamiento de la experiencia que tienen entidades tales como CONICYT, CORFO e ICM al respecto. Lo anterior, además, debería ser complementado con baterías de indicadores de resultados e impactos formulados por cada región a partir de sus estrategias y/o políticas de innovación, con metodologías claras y factibles para su medición.

Para el caso de las agencias públicas que presentan instrumentos, se hace necesario velar por la pertinencia de ellos, mediante un trabajo tanto a nivel nacional como de cada región (aprovechando para esto instancias como las que se proponen en este punto) que formule requerimientos que sean de cumplimiento obligatorio para que puedan optar a financiamiento de la provisión.

Asimismo, se propone solicitar cofinanciamiento de parte de ellas con recursos de sus propios presupuestos ${ }^{8}$ (monto o porcentaje a definir) y exigir que se contemplen instancias de participación regional dentro de la evaluación y seguimiento de la ejecución de los proyectos que se ejecuten en el marco de los instrumentos financiados.

De manera transversal, se requiere profundar en la sensibilización y capacitación de actores relevantes ligados a los procesos de operación del FIC regional, especialmente para lograr su comprensión respecto a la justificación, características y a los conceptos que están asociados a la inversión en esta materia.

En ese sentido, es importante considerar que un trabajo como el señalado requiere de capacidades especiales de los sectores público, privado y académico de las regiones, que permitan la comprensión y gestión de las fuentes y las particularidades que están asociadas a los procesos de innovación.

Relacionado con lo anterior, se sugiere mejorar los criterios con los cuales se distribuyen los recursos del FIC Regional, introduciendo otros que permitan valorar la eficiencia en el uso de los recursos, la existencia de un compromiso formal de vinculación del uso del fondo a instrumentos de planificación regional y de una gobernanza regional que sea parte clave en la labor que realiza cada región en materia de innovación y el aporte de recursos adicionales a los de la provisión a este tema (tanto públicos como privados).

En cuanto a los ejecutores, se sugiere establecer un procedimiento para la inclusión de otros adicionales a los ya existentes que contemple criterios tales como pertinencia de la acción que realiza y experiencia de ella, además de la evaluación que efectúen actores regionales ligados a la provisión.

Esto también podría aplicarse para evaluar a ejecutores actuales, de tal manera de determinar la importancia, pertinencia y la conveniencia de que permanezcan como tal, pudiendo establecer recomendaciones de carácter vinculante para condicionar su continuidad.

Por último, en relación al proceso de asignación, se hace necesario que la mayor parte de él pueda efectuarse dentro del año anterior a su inicio, de tal forma de que su ejecución pueda comenzar efectivamente dentro del año presupuestario que corresponda.

Tal como ya se mencionó, la falta de información procesada y sistematizada respecto al comportamiento que ha tenido la provisión en cada región (en términos

\footnotetext{
${ }^{8}$ Siguiendo, a modo de ejemplo, la experiencia que tiene CONICYT con la creación de un Fondo Complementario con recursos propios para cofinanciar asignaciones de recursos de la provisión.
} 
de asignación y ejecución de recursos y de resultados obtenidos) es un serio problema para efectuar estudios que permitan evaluarla, lo cual conspira en contra del interés por respaldar y potenciar esta fuente de recursos y, a la vez, instrumento de política pública. Esto no implica asumir un control o tutela de la acción que los gobiernos regionales realizan en materia de innovación, sino que se orienta a contar con una instancia que tenga el rol de articular un trabajo conjunto entre ellos para promover el intercambio de experiencias y aprendizajes y la realización de proyectos conjuntos de mutuo interés y utilidad, además de aunar información que permita efectuar análisis que sirvan para visibilizar la relevancia y resultados que se han obtenido en actores relevantes, así como también generar insumos para justificar su ampliación y mejoramiento (tales como evaluaciones de resultados e impactos, entre otras).

En ese sentido, se recomienda que sea SUBDERE quien asuma ese rol, mediante la realización de un trabajo que trate de manera separada, pero coordinada, aspectos de políticas regionales de innovación y mejoramiento de la competitividad con aspectos de inversión pública regional en dicha materia. Esto considerando que dos de los principales roles de los gobiernos regionales dicen relación con el diseño e implementación de instrumentos de planificación regional y con la realización de inversiones que sean coherentes con ellos. Este trabajo también podría enriquecerse con la generación de una instancia que permita la articulación con organismos públicos, académicos, privados y no gubernamentales ligados al tema.

Además, se recomienda aprovechar la oportunidad que entrega la plataforma Chile Indica para el registro de la información de la acción que cada gobierno regional realiza en dicha materia, ya que esta herramienta tiene un grado de posicionamiento dentro de los gobiernos regionales, lo que permitiría reducir costos y tiempos de adaptación e instalación de procedimientos. En cuanto a los mecanismos, se sugiere evaluar opciones tales como inclusión de condiciones dentro de las glosas comunes del presupuesto de los gobiernos regionales $\mathrm{y} / \mathrm{u}$ otras similares.

\section{BIBLIOGRAFÍA}

CONICYT (2015). Principales indicadores cienciométricos de la actividad científica chilena 2013. Trabajo realizado en conjunto con Scimago.

Balbontín R., Roeschmann J. y Zahler A. (2018). Ciencia, Tecnología e Innovación en Chile: un análisis presupuestario. Serie de Estudios de Finanzas Públicas, DIPRES.

Crespi G., Fernández-Arias E. y Stein E. (2014). ¿Cómo repensar el desarrollo productivo?: políticas e instituciones sólidas para la transformación económica. Banco Interamericano de Desarrollo.

DIPRES (2011). Programa Provisión Fondo de Innovación para la Competitividad (FIC). Informe final. Evaluación de programas gubernamentales (EPG).

Eyzaguirre N., Marcel M., Rodríguez J. y Tokman M. (2005). Hacia la economía del conocimiento: el camino para crecer con equidad en el largo plazo. Revista Estudios Públicos, 97. Verano 2005.

Ministerio de Economía (2016a). Resultados preliminares V Encuesta Nacional sobre Gasto y Personal en I+D. Presentación en diapositivas. 
(2016b). 9na. Encuesta de innovación en empresas (2013-2014). Equipo Inteligencia de Datos, División de Innovación. Presentación en diapositivas. (2012). 7ma. Encuesta de innovación en empresas, 2009-2010. Principales Resultados. Gobierno de Chile. (2010). Resultados de las Encuestas de Innovación e I+D 2007-2008. División de Innovación. Presentación en diapositivas.

Lauterbach R. (2015), Chile y la complejidad de sus exportaciones. Serie de documentos de trabajo $\mathrm{N}^{\circ}$. Consejo Nacional de Innovación para el Desarrollo.

SUBDERE (2011). Aprueba procedimientos y modalidades de aplicación de la provisión Programa Fondo de Innovación para la Competitividad conforme a lo dispuesto en la glosa $\mathrm{N}^{\circ} 15$ del programa 01 del presupuesto de la Subsecretaría de Desarrollo Regional y Administrativo correspondiente al año 2012. Resolución afecta $\mathrm{N}^{\circ} 277$, de fecha 30 de diciembre. Tomada de razón con fecha 1 de marzo de 2012.

(2016). Sustituye texto de resolución $\mathrm{N}^{\circ} 277$, de 2011, de esta Subsecretaría, sobre procedimientos y modalidades de aplicación del Fondo de Innovación para la Competitividad. Resolución afecta $\mathrm{N}^{\circ} \mathrm{O} 2$, de fecha 14 de enero. Tomada de razón con fecha 7 de noviembre de 2016.

\section{SITIOS WEB}

SUBDERE (2017). Estadísticas de SUBDERE respecto a la asignación y ejecución de recursos de la provisión FIC. Extraídas desde http://www.chileindica.cl/

UNED (2016). Resumen del Manual de Oslo sobre Innovación. Extraído desde http://portal.uned.es/portal/page?_pageid=93,23280929\&_dad=portal, visitado el 31 de agosto de 2016. 


\section{ANEXO A: OTRAS CARACTERÍSTICAS DE LA PROVISIÓN FIC-R}

\begin{tabular}{|c|c|}
\hline Hitos importantes & $\begin{array}{l}\text { Ingreso de bases de concursos para entidades receptoras a } \\
\text { SUBDERE: hasta el } 30 \text { de abril de cada año (en la resolución } \\
\mathrm{N}^{\circ} 277 / 2011 \text { este plazo era hasta el } 31 \text { de marzo). } \\
\text { Asignación de recursos a agencias ejecutoras y corporaciones } \\
\text { de desarrollo: hasta el } 31 \text { de mayo de cada año (en la } \\
\text { resolución } \mathrm{N}^{\circ} 277 / 2011 \text { este plazo era hasta el } 30 \text { de junio). }\end{array}$ \\
\hline $\begin{array}{l}\text { Otras } \\
\text { características }\end{array}$ & $\begin{array}{l}\text { La evaluación de los proyectos presentados mediante la } \\
\text { convocatoria realizada para entidades receptoras debía ser } \\
\text { efectuada por una comisión conformada por } 2 \text { representantes } \\
\text { del sector empresarial, } 2 \text { representantes de agencias } \\
\text { ejecutoras y/o de corporaciones de desarrollo y, } 2 \\
\text { funcionarios del servicio administrativo del gobierno regional } \\
\text { (SAGORE), todos los cuales deberán cumplir con los } \\
\text { requisitos que establezca la resolución } \mathrm{N}^{\circ} 277 \text {, 2011 de } \\
\text { SUBDERE. No obstante, la resolución } \mathrm{N}^{\circ} \text {, 2016 de } \\
\text { SUBDERE modificó eso, permitiendo que la evaluación sea } \\
\text { efectuada por un panel externo de expertos que contraten } \\
\text { cada gobierno regional para ese fin, o bien, mediante la } \\
\text { constitución de una comisión técnica de evaluación que } \\
\text { deberá estar integrada, a lo menos, por } 2 \text { representantes del } \\
\text { sector empresarial y } 2 \text { funcionarios del SAGORE, debiendo al } \\
\text { menos uno de ellos cumplir tareas ligadas a la política y/o } \\
\text { estrategia regional de innovación. } \\
\text { Entrelos años } 2011 \text { y } 2014 \text { (ambos inclusive) se destinó un } 5 \% \\
\text { del total de la provisión para el fortalecimiento de las } \\
\text { capacidades de los gobiernos regionales y de la } \\
\text { institucionalidad pública o privada para el desarrollo regional } \\
\text { de la innovación, los cuales fueron administrados por } \\
\text { SUBDERE. Con cargo a estos recursos se financiaron } \\
\text { iniciativas tales como diplomados de capacitación y } \\
\text { contratación de acompañamiento experto para la elaboración } \\
\text { de estrategias regionales de innovación (ERI), entre otras. } \\
\text { La resolución No } 277,2011 \text { de SUBDERE, contempla la } \\
\text { posibilidad de redistribuir los recursos que no hayan } \\
\text { comprometido los gobiernos regionales dentro de los plazos } \\
\text { señalados anteriormente, o bien, asignarlos mediante un } \\
\text { concurso nacional. } \\
\text { La glosa presupuestaria asociada a la provisión, para el año } \\
2010, \text { contempló la posibilidad de que los gobiernos } \\
\text { regionales propusieran el ingreso de otras agencias ejecutoras } \\
\text { públicas o privadas, lo cual debía ser evaluado por el } \\
\text { Ministerio de Economía y aprobado por el Comité de } \\
\text { Ministros para la Innovación. }\end{array}$ \\
\hline
\end{tabular}




\begin{tabular}{|c|c|}
\hline & $\begin{array}{l}\text { La resolución } \mathrm{N}^{\circ} 2,2016 \text {, incluyó una serie de nuevas agencias } \\
\text { ejecutoras, entre las cuales está SERCOTEC y ProChile. } \\
\text { Además, incluyó los comités de fomento productivo } \\
\text { regionales. También amplió el abanico de entidades } \\
\text { receptoras, permitiendo el ingreso de centros regionales de } \\
\text { CONICYT y de incubadoras de negocios de innovación de } \\
\text { CORFO. } \\
\text { A partir de la resolución } \mathrm{N}^{\circ} 2,2016 \text {, las entidades receptoras } \\
\text { privadas deberán aportar el } 10 \% \text { del costo tal de la iniciativa, } \\
\text { debiendo ser la mitad aporte pecuniario. }\end{array}$ \\
\hline $\begin{array}{l}\text { Principales } \\
\text { comentarios } \\
\text { DIPRES a partir } \\
\text { de evaluación } \\
\text { efectuada a la } \\
\text { provisión }\end{array}$ & $\begin{array}{l}\text { La existencia de la provisión está justificada, ya que la } \\
\text { necesidad que le dio origen aún existe. } \\
\text { El diseño del programa es adecuado, no obstante, recomienda } \\
\text { profundizar en materia de ampliación de competencias y } \\
\text { generación de redes regionales de cooperación, así como } \\
\text { también de gobernabilidad y conducción estratégica de } \\
\text { investigación, desarrollo e innovación (I+D+i). Además, } \\
\text { recomienda dar un carácter plurianual al financiamiento, } \\
\text { dado que corresponde más a las características propias de los } \\
\text { proyectos de I+D+i. } \\
\text { Se propone: } \\
\text { Mejorar el seguimiento de proyectos, así como también de su } \\
\text { reportabilidad técnica y financiera asociada. Esto tanto a nivel } \\
\text { central como regional. } \\
\text { Revisar prácticas centralistas, tales como la revisión de } \\
\text { pertinencia de los proyectos desde el nivel central, mejorando } \\
\text { su transparencia y claridad, y evaluado } \\
\text { complementariedad mediante el aprovechamiento de } \\
\text { capacidades e instancias que las regiones disponen. } \\
\text { La evaluación de proyectos podría efectuarse mediante la } \\
\text { constitución de redes regionales de especialistas en temas } \\
\text { priorizados por los gobiernos regionales. } \\
\text { Promover la complementariedad de los recursos de la } \\
\text { provisión con otras fuentes de financiamiento, tanto } \\
\text { nacionales y regionales. } \\
\text { Revisar la forma de distribución regional de los recursos de la } \\
\text { provisión. } \\
\text { Promover de parte de las agencias ejecutoras, la aplicación de } \\
\text { los recursos con base en necesidades regionales y no con las } \\
\text { limitaciones que regulan los instrumentos. } \\
\text { Revisar el procedimiento de asignación de recursos de parte } \\
\text { de cada gobierno regional, de tal manera de adelantarlo y } \\
\text { reducir sus plazos. }\end{array}$ \\
\hline
\end{tabular}

Fuente: elaboración propia con base en SUBDERE, 2011, SUBDERE, 2016 y DIPRES, 2011. 
Revista LIDER, 36(22), pp. 56-88

Como antecedente adicional, es importante señalar que la Contraloría General de la República ha efectuado auditorías financieras a la provisión FIC a diversos organismos ligados a ella (tales como SUBDERE, Subsecretaría de Economía, CORFO, CONICYT y gobiernos regionales). Para el caso de SUBDERE, el informe final data del 1 de abril de 2013. 